\title{
Ambiguity Aversion with Three or More Outcomes
}

\author{
By Mark J. MachinA*
}

\begin{abstract}
Ambiguous choice problems which involve three or more outcome values can reveal aspects of ambiguity and ambiguity aversion which cannot be displayed in the classic two-outcome Ellsberg urn problems, and hence are not always captured by models designed to accommodate them. These aspects include Allais-type preferences over purely subjective acts, attitudes toward different sources involving different amounts of ambiguity, and attitudes toward ambiguity at different outcome levels. This paper presents a few such examples, and examines the standard models' predictions and performance in such cases. (JEL D81)
\end{abstract}

Three Decision Problems.-Consider the following decision problems. The Slightly-Bent Coin Problem involves two sources of ambiguity. One source is a coin which has been slightly bent in an unknown direction. It still has well-defined probabilities, in the sense that if it were to be flipped millions of times, there is some fixed value to which the proportion of heads would converge-you just don't know what that value is, and you only get to flip once. In this sense it exhibits exactly the same type of ambiguity as displayed by the event black (or yellow) in the classic Three-Color Ellsberg Urn-repeated sampling with replacement would also yield some fixed limiting proportion of black draws, but again, you don't know what that proportion is. The only difference is that since the coin is only slightly bent, you know that its unknown proportion is very close to one-half. The other source of ambiguity in the problem is an urn containing a ball, which could be either black or white. The mechanics of the coin flip does not depend upon the contents of the urn, and the coin is flipped and the ball drawn simultaneously. The bets are based on the outcome of the flip and the color of the ball.

The next problem, the Thermometer Problem, involves bets on the temperature in Timbuktu at noon next May Day. The thermometer is more than just a very accurate digital thermometer-it's a perfectly accurate analog thermometer, which can exactly report any value in the continuum. Divide the continuum of feasible temperatures into

\footnotetext{
* Department of Economics, University of California-San Diego, 9500 Gilman Dr., La Jolla, CA 92093 (e-mail: mmachina@ucsd.edu). I am grateful to Najeeb Ali, Jim Andreoni, Aurélian Baillon, Guillaume Carlier, Rose-Anne Dana, David Dillenberger, Mark Durst, Daniel Ellsberg, Larry Epstein, Paolo Ghirardato, Itzhak Gilboa, Mark Jacobsen, Edi Karni, Peter Klibanoff, Olivier L'Haridon, Robert Nau, Lætitia Placido, Ben Polak, Jacob Sagi, Martin Schonger, Uzi Segal, Marciano Siniscalchi, Jiankang Zhang, and especially Alain Chateauneuf, Chris Chambers, Joel Sobel, Peter Wakker, and anonymous referees for helpful comments. All errors and opinions are mine. Financial support from the Center for the Economic Analysis of Risk (CEAR) at Georgia State University is gratefully acknowledged. The author declares that he has no relevant or material financial interests that relate to the research described in this paper.

${ }^{\dagger}$ Go to http://dx.doi.org/10.1257/aer.104.12.3814 to visit the article page for additional materials and author disclosure statement(s).
} 
Slightly-Bent Coin Problem

\begin{tabular}{|c|c|c|c|c|c|c|}
\hline & \multicolumn{2}{|c|}{ Bet I } & & & \multicolumn{2}{|c|}{ Bet II } \\
\hline & Black & White & & & Black & White \\
\hline Heads & $+\$ 8,000$ & $\$ 0$ & & Heads & $\$ 0$ & $\$ 0$ \\
\hline Tails & $-\$ 8,000$ & $\$ 0$ & versus & Tails & $-\$ 8,000$ & $+\$ 8,000$ \\
\hline
\end{tabular}

Thermometer Problem

\begin{tabular}{lll}
\hline \hline Bet 1 (\$6K if $t$ in left $45 / 100$ th of any interval) & versus & Bet $2(\$ 3 \mathrm{~K}$ if $t$ in left $90 / 100$ th of any interval $)$ \\
Bet $3(\$ 6 \mathrm{~K}$ if $t$ in left $1 / 1000$ th of any interval) & versus & Bet $4(\$ 3 \mathrm{~K}$ if $t$ in left $2 / 1000$ th of any interval $)$ \\
\hline
\end{tabular}

Ambiguity at Low versus High Outcomes Problem-Three-Color Version

$(\$ c=$ Your certainty equivalent of the objective bet $(\$ 0,1 / 2 ; \$ 100,1 / 2))$

\begin{tabular}{|c|c|c|c|c|c|c|c|}
\hline & & \multirow{2}{*}{$b_{0}$} & 1 ball & 1 ball & 1 ball & & \\
\hline & & & $\$ 0$ & $\$ c$ & $\$ 100$ & & \\
\hline & Urn I & & & & \multicolumn{3}{|c|}{ Urn II } \\
\hline \multicolumn{2}{|c|}{2 balls } & & 1 ball & \multirow{3}{*}{ versus } & 1 ball & \multicolumn{2}{|c|}{2 balls } \\
\hline Black & White & & Red & & Red & Black & White \\
\hline$\$ 0$ & $\$ c$ & & $\$ 100$ & & $\$ 0$ & $\$ c$ & $\$ 100$ \\
\hline
\end{tabular}

an extremely large number of equal-length intervals. Bet 1 yields its prize of $\$ 6,000$ if the temperature $t$ lands in the left 45/100th portion of any interval and $\$ 0$ if it lands in the right 55/100th portion, and Bets 2, 3, and 4 have a similar structure.

The final problem, which we call the Ambiguity at Low versus High Outcomes Problem, consists of a choice between bets on two Ellsberg-type urns. Each bet can be contrasted with the purely objective bet $b_{0}=\left(\$ 0, \frac{1}{3} ; \$ c, \frac{1}{3} ; \$ 100, \frac{1}{3}\right)$, where $\$ c$ is your certainty equivalent of an objective $50: 50$ bet for $\$ 0$ or $\$ 100$. Urn I differs from the purely objective bet $b_{0}$ in having ambiguity across its lower and middle outcome values $\$ 0$ and $\$ c$, whereas Urn II differs from $b_{0}$ in having ambiguity across its middle and upper outcome values $\$ c$ and $\$ 100$.

I.

The concept of objective uncertainty dates back at least to seventeenth century French gamblers such as Pascal and Fermat, and mathematicians have since developed the theory of probability far beyond what is needed (or could even be applied) by economists or other decision theorists. Although humans faced situations of subjective uncertainty (plagues, earthquakes ...) long before the invention of dice or roulette wheels, the formal recognition and specification of subjective uncertainty as a distinct concept is much more recent. ${ }^{1}$ More recent still is the formal development 
of subjective probability, in which the theory of probability can be applied to an individual's beliefs - and hence their decisions - in subjective settings, and which has typically been posited jointly with expected utility risk preferences and termed subjective expected utility. ${ }^{2}$

While the combination of subjective probability theory with classical expected utility theory would seem to constitute the ideal framework for the analysis of choice under uncertainty, a still more recent phenomenon has caused researchers to question the empirical validity of the subjective probability hypothesis. These are the well-known thought experiments proposed by Daniel Ellsberg (1961). So-called Ellsberg urns present situations of objective, subjective and mixed objective/subjective uncertainty, and most individuals' preferences for bets on such urns seem systematically to violate the existence of well-defined subjective probabilities. This feature of preferences has been termed ambiguity aversion. Economists and others have responded to this phenomenon by developing models-typically generalizations of subjective expected utility - designed to accommodate ambiguity aversion, and such models have been usefully applied to the analysis of economic behavior.

Although the major models of ambiguity aversion are of course defined over multiple-outcome prospects, the thought experiments in Ellsberg's classic 1961 article only involved bets over a single pair of outcome values (Ellsberg used $\$ 0$ and \$100). But even in the world of objective uncertainty, bets on just a single pair of outcome values - that is, a family of objective lotteries of the form $\{(\$ 100, p ; \$ 0,1-p) \mid p \in[0,1]\}$ - cannot reveal much about an individual's attitudes toward risk, not even whether they are risk-neutral, risk-averse or risk-loving.

Ellsberg's two-outcome, mixed-uncertainty examples did a little better, in allowing an individual's aversion or preference for ambiguity to reveal itself, and the major models of ambiguity aversion have provided different ways to represent this. However, just as with the objective case, there are aspects of ambiguity and ambiguity aversion which can only reveal themselves in three-or-more-outcome choice problems, such as the ones presented at the beginning of this paper. ${ }^{3}$

The purpose of this paper is to consider three such aspects. Since the major models were motivated by and designed to accommodate Ellsberg's classic two-outcome examples, it is perhaps no surprise that in most cases they predict neutrality toward these new aspects. This too has an analogue in the objective case: preferences over the two-outcome family $\{(\$ 100, p ; \$ 0,1-p) \mid p \in[0,1]\}$ can be successfully modeled by the hypothesis of expected value maximization, but this hypothesis would predict neutrality toward aspects of risk and risk aversion which can arise once three or more outcomes are allowed.

The following section reviews the classic Ellsberg urn examples and some of the models which have been developed in response to them. Sections III, IV, and V

\footnotetext{
${ }^{2}$ See, e.g., Savage (1954); Anscombe and Aumann (1963).

${ }^{3}$ Ellsberg did examine some three-outcome examples as part of his critique of the Sure-Thing Principle (e.g., Ellsberg 2001, pp.148, 152-54, 189, 208, 215, 225), but these seem to have escaped the attention of modern researchers. Three-outcome examples involving subjective uncertainty also appear in Luce and Raiffa (1957, chapter 13); Nau (2001, 2006); Zhang (2002); Lo (2008); Ergin and Gul (2009); and Wakker (2010), and have been experimentally examined by MacCrimmon and Larsson (1979, section 8); Tversky and Kahneman (1992); Fennema and Wakker (1996); Wu and Gonzalez (1999); Diecidue, Wakker, and Zeelenberg (2007); L'Haridon and Placido (2010); and Hey, Lotito, and Maffioletti (2010). However, to my knowledge none of the issues addressed in this paper have appeared in the literature.
} 
Three-Color Ellsberg Paradox

\begin{tabular}{rrrrrr}
\hline \hline & $\overbrace{\text { Red }}^{30 \text { balls }}$ & \multicolumn{3}{c}{60 balls } \\
\cline { 4 - 5 }$a_{1}$ & $\$ 100$ & & $\begin{array}{c}\text { Black } \\
\$ 0\end{array}$ & Yellow & \\
$a_{2}$ & $\$ 0$ & & $\$ 100$ & $\$ 0$ & $\curlyvee$ \\
$a_{3}$ & $\$ 100$ & & $\$ 0$ & $\$ 100$ & \\
$a_{4}$ & $\$ 0$ & $\$ 100$ & $\$ 100$ & $\curlywedge$ \\
\hline
\end{tabular}

present aspects of ambiguity and ambiguity aversion which can arise in a world with three or more outcomes, and examine how the major models perform in such cases. Section VI concludes.

\section{Classic Urns and Major Models}

\section{A. Classic Ellsberg Urn Problems}

In his 1961 article and $1962 \mathrm{PhD}$ thesis, ${ }^{4}$ Ellsberg presented a class of decision problems involving both subjective and objective uncertainty, which seem to contradict the classic subjective expected utility hypothesis as formalized and axiomatized in Savage (1954). The example now known as the Three-Color Ellsberg Paradox involves an opaque urn containing 90 balls. Exactly 30 of these balls are known to be red, and each of the other 60 is either black or yellow, but the exact numbers are unknown, and could be anywhere from 0:60 to 60:0. A ball is to be drawn from the urn, and the decision maker is presented with two pairs of bets based on the color of the ball, as illustrated above. 5

Ellsberg conjectured, and subsequent experimenters have found, ${ }^{6}$ that most individuals would prefer bet $a_{1}$ over bet $a_{2}$, and bet $a_{4}$ over bet $a_{3}$, which we will refer to as Ellsberg preferences in this choice problem. The example is termed a paradox since such preferences directly (and systematically) contradict the subjective probability hypothesis - if the individual did assign subjective probabilities to the events \{red, black, yellow\}, then the strict preference ranking $a_{1} \succ a_{2}$ would reveal the strict subjective likelihood ranking prob(red) $>\operatorname{prob}$ (black), but the ranking $a_{3} \prec a_{4}$ would reveal prob(red) $<$ prob(black).

The widely accepted reason for such preferences is that while the bet $a_{1}$ guarantees a known probability $1 / 3$ of winning the $\$ 100$ prize, the probability of winning offered by $a_{2}$ is unknown, and could be anywhere from 0 to $2 / 3$. Although this range has $1 / 3$ as its midpoint, and there is no reason to expect any asymmetry, individuals seem to prefer the known to the unknown probability. Similarly, bet $a_{4}$ offers a guaranteed $2 / 3$ chance of winning, whereas the probability offered by $a_{3}$ could be anywhere from $1 / 3$

\footnotetext{
${ }^{4}$ Ellsberg's thesis has since been published as Ellsberg (2001).

${ }^{5}$ Ellsberg (1961, pp. 653-56; 2001, pp. 42-45, 155-58). Ellsberg (2001, pp. 137) gives an equivalent version with the payoffs $\$ 100$ : $\$ 0$ replaced by $\$ 0:-\$ 100$. Ellsberg $(1961$, p. 651, 659; 2001, pp. 43-44, 136-37, 201-02) refers to similar work by Chipman (1960) (see also Chipman 1958). Ellsberg reminisces about his earlier views on the subject in Ellsberg (2001, p. 244, footnote 2).

${ }^{6}$ See the surveys of Camerer and Weber (1992); Kelsey and Quiggin (1992); Camerer (1995); Siniscalchi (2008a); Al-Najjar and Weinstein (2009); Hey, Lotito, and Maffioletti (2010); Etner, Jeleva, and Tallon (2012); Gilboa and Marinacci (2013); and Machina and Siniscalchi (2014).
} 
Two-Urn Ellsberg Paradox

\begin{tabular}{|c|c|c|c|c|}
\hline & \multicolumn{2}{|c|}{ Urn I } & \multicolumn{2}{|c|}{ Urn II } \\
\hline & \multicolumn{2}{|c|}{100 balls } & 50 balls & 50 balls \\
\hline & Red & Green & Black & Yellow \\
\hline$b_{1}$ & $\$ 100$ & $\$ 0$ & & \\
\hline$b_{2}$ & & & $\$ 100$ & $\$ 0$ \\
\hline$b_{3}$ & $\$ 0$ & $\$ 100$ & & \\
\hline$b_{4}$ & & & $\$ 0$ & $\$ 100$ \\
\hline
\end{tabular}

Four-Color Ellsberg Paradox

\begin{tabular}{rrrrr}
\hline \multicolumn{4}{c}{$($ Single urn $)$} \\
& $\overbrace{\text { Red }}^{100 \text { balls }}$ & Green & $\overbrace{\text { Black }}^{50 \text { balls }}$ & $\overbrace{\text { Yellow }}^{50 \text { balls }}$ \\
$c_{1}$ & $\$ 100$ & $\$ 100$ & $\$ 0$ & $\$ 0$ \\
$c_{2}$ & $\$ 100$ & $\$ 0$ & $\$ 100$ & $\$ 0$ \\
$c_{3}$ & $\$ 0$ & $\$ 100$ & $\$ 0$ & $\$ 100$ \\
$c_{4}$ & $\$ 0$ & $\$ 0$ & $\$ 100$ & $\$ 100$
\end{tabular}

to 1. Again, individuals prefer the known-probability bet. Ellsberg referred to bets $a_{2}$ and $a_{3}$ as involving ambiguity, and a preference for known-probability over ambiguous bets has come to be known as ambiguity aversion.

Ellsberg's article contained two additional widely cited examples. In the left-hand example above, known as the Two-Urn Ellsberg Paradox,,$^{7}$ Urn I contains 100 red and green balls in unknown proportions, and Urn II contains exactly 50 black and 50 yellow balls. Again, typical preferences are for the known-probability bets $b_{2}$ and $b_{4}$ over their unknown-probability counterparts $b_{1}$ and $b_{3}$, again contradicting the subjective probability hypothesis, ${ }_{\square}^{8}$ and reflecting the same type of ambiguity aversion as in the Three-Color Paradox. In the right-hand example, suggested to Ellsberg by Kenneth Arrow and known as the Four-Color Ellsberg Paradox, ${ }^{9}$ the typical rankings $c_{1} \succ c_{2}$ and $c_{3} \prec c_{4}$ imply prob(green) $>$ prob(black) and $\operatorname{prob}($ green $)<\operatorname{prob}($ black $)$, respectively, and reveal the same type of ambiguity aversion as in the previous examples. Ellsberg observed that such examples can be viewed as providing systematic violations of Savage's Sure-Thing Principle, often termed the property of event-separability. ${ }^{10}$ As mentioned, such examples have received a great deal of experimental confirmation (see footnotes 3 and 6).

\section{B. Major Models of Ambiguity Aversion}

Ellsberg's examples have spurred the development of several models which generalize the classic Subjective Expected Utility Model to allow for ambiguity aversion. In the finite-outcome setting of this paper, the objects of choice consist of purely objective lotteries $\mathbf{P}=\left(x_{1}, p_{1} ; \ldots ; x_{n}, p_{n}\right)$ yielding outcome $x_{j}$ with probability $p_{j}$ over some (say, monetary) outcome set $\mathcal{X}$, purely subjective acts $f(\cdot)$ $=\left[x_{1}\right.$ on $E_{1} ; \ldots ; x_{n}$ on $\left.E_{n}\right]$ yielding $x_{i}$ on event $E_{i}$ for some partition $\left\{E_{1}, \ldots, E_{n}\right\}$ of a subjective state space $\mathcal{S}=\{\ldots, s, \ldots\}$ or $\mathcal{S} \subseteq \mathbf{R}^{\mathbf{N}}$, and mixed objective/subjective bets $^{11}\left[\mathbf{P}_{1}\right.$ on $E_{1} ; \ldots ; \mathbf{P}_{n}$ on $\left.E_{n}\right]$, which are subjective bets whose outcomes consist of

\footnotetext{
${ }^{7}$ Ellsberg (1961, pp. 650-53).

${ }^{8} b_{1} \prec b_{2}$ would reveal prob(red) $<\operatorname{prob}($ black$)$, but $b_{3} \prec b_{4}$ would reveal prob(green) $<\operatorname{prob}($ yellow), violating the requirement that $\operatorname{prob}($ red $)+\operatorname{prob}($ green $)=\operatorname{prob}($ black $)+\operatorname{prob}($ yellow $)=1$.

${ }^{9}$ Ellsberg (1961, p. 654, footnote 4). Ellsberg offers another variation in (1961, p. 651, footnote 1).

${ }^{10}$ Savage (1954, p. 23, postulate P2). Since these examples also violate Axiom P4* (Strong Comparative Probability) of Machina and Schmeidler (1992, p. 761), they also violate the more general property of probabilistic sophistication (1992, pp. 753, 755).

${ }^{11}$ Such bets are also known as Anscombe-Aumann acts (Anscombe and Aumann 1963). To demonstrate that the issues discussed in this paper are not due to two-stage resolution of uncertainty, all mixed prospects are assumed to involve simultaneous resolution of both sources of uncertainty.
} 
objective lotteries $P_{i}=\left(\ldots ; x_{i j}, p_{i j} ; \ldots\right)$. The family of mixed objective/subjective bets is seen to include the family of purely objective lotteries and the family of purely subjective acts.

Classical Subjective Expected Utility (SEU) preferences over such prospects can be represented by an ordinal preference function of the form

$$
W_{S E U}\left(\ldots ; x_{i} \text { on } E_{i} ; \ldots\right)=\sum_{i} U\left(x_{i}\right) \cdot \pi\left(E_{i}\right)
$$

over purely subjective acts, and more generally, the form

$$
W_{S E U}\left(\ldots ;\left(\ldots ; x_{i j}, p_{i j} ; \ldots\right) \text { on } E_{i} ; \ldots\right)=\sum_{i}\left[\sum_{j} U\left(x_{i j}\right) \cdot p_{i j}\right] \cdot \pi\left(E_{i}\right)
$$

over mixed objective/subjective bets, for some increasing cardinal utility function $U(\cdot)$ over outcomes and additive subjective probability measure (prior) $\pi(\cdot)$ over events.

One of the major models of ambiguity aversion over subjective or mixed objective/subjective bets-formalized by Gilboa and Schmeidler (1989) and termed the Expected Utility with Multiple Priors (or simply Multiple-Priors) Model-captures ambiguity aversion by means of the form

$$
W_{M P}\left(\ldots ; x_{i} \text { on } E_{i} ; \ldots\right)=\min _{\pi \in \Pi_{0}} \sum_{i} U\left(x_{i}\right) \cdot \pi\left(E_{i}\right)
$$

over purely subjective acts, and more generally

$$
W_{M P}\left(\ldots ;\left(\ldots ; x_{i j}, p_{i j} ; \ldots\right) \text { on } E_{i} ; \ldots\right)=\min _{\pi \in \Pi_{0}} \sum_{i}\left[\sum_{j} U\left(x_{i j}\right) \cdot p_{i j}\right] \cdot \pi\left(E_{i}\right)
$$

for some family $\Pi_{0}$ of subjective probability measures $\pi(\cdot)$ over events. 12 The intuition behind this form is that an ambiguity averter would evaluate each subjective or mixed bet in the most pessimistic way, given their family of priors $\Pi_{0}$.

A second important model of ambiguity aversion, the Rank-Dependent (or Choquet) Model of Schmeidler (1989), ${ }^{13}$ which takes the form

$$
W_{R D}\left(\ldots ; x_{i} \text { on } E_{i} ; \ldots\right)=\sum_{i} U\left(x_{i}\right) \cdot\left(C\left(\cup_{k=1}^{i} E_{k}\right)-C\left(\cup_{k=1}^{i-1} E_{k}\right)\right)
$$

over purely subjective acts, and more generally

$$
\begin{aligned}
& W_{R D}\left(\ldots ;\left(\ldots ; x_{i j}, p_{i j} ; \ldots\right) \text { on } E_{i} ; \ldots\right) \\
& \quad=\sum_{i}\left[\sum_{j} U\left(x_{i j}\right) \cdot p_{i j}\right] \cdot\left(C\left(\cup_{k=1}^{i} E_{k}\right)-C\left(\cup_{k=1}^{i-1} E_{k}\right)\right)
\end{aligned}
$$

\footnotetext{
${ }^{12}$ To avoid technical issues, throughout this paper we restrict attention to families of priors $\Pi_{0}$ (and below, to probability measures $\mu(\cdot))$ with closure, boundary and/or continuity properties sufficient to ensure that the minima in equations such as (3), (4), (7), (11), and (12), and integrals in equations such as (9) and (10), exist and are finite.

${ }^{13}$ See also Gilboa (1987), which derives from an earlier version of Schmeidler (1989).
} 
over mixed objective/subjective bets, for some nonadditive measure (capacity) $C(\cdot)$, and where in (5) the outcomes $x_{i}$ and their corresponding events $E_{i}$ are labeled so that $x_{1} \succcurlyeq \ldots \succcurlyeq x_{n}$, and in (6) the conditional lotteries $\left(\ldots ; x_{i j}, p_{i j} ; \ldots\right)$ and events $E_{i}$ are labeled so that the conditional expected utility values satisfy $\sum_{j} U\left(x_{1 j}\right) \cdot p_{1 j} \geq \cdots \geq \sum_{j} U\left(x_{n j}\right) \cdot p_{n j} \cdot{ }^{14}$ The intuition behind the use of a nonadditive measure is that the union of two ambiguous events (such as black and yellow in the Three-Color Urn) could well be purely objective, and it requires a nonadditive measure $C(\cdot)$ over events to capture the preference implications of this. The event $\cup_{k=1}^{i} E_{k}$ on which an outcome of at least $x_{i}$ is received is sometimes referred to as the bet's good-news event for the outcome level $x_{i}$.

The Rank-Dependent form (5) has two important special cases, corresponding to different properties of the capacity $C(\cdot) . C(\cdot)$ is said to be convex if it satisfies $C(\hat{E} \cup E)-C(E) \geq C(\hat{E})-C(\hat{E} \cap E)$ for all events $E$ and $\hat{E} .^{15}$ In such a case, (5) can also be expressed in the form

$$
W_{R D \text { convex }}\left(\ldots ; x_{i} \text { on } E_{i} ; \ldots\right)=\min _{\pi \in \Pi_{0}} \sum_{i} U\left(x_{i}\right) \cdot \pi\left(E_{i}\right):
$$

that is, as an example of the Multiple-Priors form (3). ${ }^{16}$ Alternatively, a capacity is said to be a distortion of a probability measure (base measure) $\pi(\cdot)$ if it takes the form $C(E)=G(\pi(E))$ for some increasing continuous distortion function $G(\cdot):[0,1] \rightarrow[0,1]$ over probabilities: ${ }^{17}$

$$
\begin{aligned}
W_{\text {RDdistortion }}\left(\ldots ; x_{i} \text { on } E_{i} ; \ldots\right) & =\sum_{i} U\left(x_{i}\right) \cdot\left(G\left(\pi\left(\cup_{k=1}^{i} E_{k}\right)\right)-G\left(\pi\left(\cup_{k=1}^{i-1} E_{k}\right)\right)\right) \\
& =\sum_{i} U\left(x_{i}\right) \cdot\left(G\left(\sum_{k=1}^{i} \pi\left(E_{k}\right)\right)-G\left(\sum_{k=1}^{i-1} \pi\left(E_{k}\right)\right)\right) .
\end{aligned}
$$

These two special cases intersect: When $G(\cdot)$ is a convex function, $C(\cdot)=G(\pi(\cdot))$ can be seen to be a convex capacity, and $W_{R D \text { distortion }}(\cdot)$ can accordingly be represented in the Multiple-Priors form (3). Properties of these special cases will matter for the results of Section III, though not for Sections IV or V.

A more recently proposed model is the Smooth Ambiguity Preferences Model of Klibanoff, Marinacci, and Mukerji $(2005,2012),{ }^{18}$ developed in part to eliminate the kinks at certainty property of the Multiple-Priors and related forms. This model takes the form

\footnotetext{
${ }^{14}$ The conditional expected utility term $\sum_{j} U\left(x_{i j}\right) \cdot p_{i j}$ in $(6)$ appears as $u(f(\cdot))$ in Schmeidler's theorem $(1989$, pp. 578-79).

${ }^{15}$ Informally, $C(\cdot)$ is convex if "adding" $\hat{E}-E$ to the event $E$ has at least as great an effect upon $C(\cdot)$ as adding $\hat{E}-E$ to the smaller event $\hat{E} \cap E$. Convexity is equivalent to Schmeidler's (1989, pp. 582-84) definition of ambiguity aversion (he uses the term uncertainty aversion).

${ }^{16}$ Schmeidler (1989, pp. 582-83). See also Schmeidler (1986, p. 260).

${ }^{17}$ See, e.g., Carlier and Dana (2003). Wakker (1990) and Chateauneuf $(1991,1994)$ have shown that a necessary and sufficient condition for a rank-dependent preference function to take this form for some $\pi(\cdot)$ and $G(\cdot)$ is that it satisfy $f(\cdot)=\left[x_{1}\right.$ if $E_{1} ; \ldots ; x_{n}$ if $\left.E_{n}\right] \succcurlyeq \hat{f}(\cdot)=\left[\hat{x}_{1}\right.$ if $\hat{E}_{1} ; \ldots ; \hat{x}_{\hat{n}}$ if $\left.\hat{E}_{\hat{n}}\right]$ whenever $\pi(\{s \in \mathcal{S} \mid f(s) \succcurlyeq x\})$ $\geq \pi(\{s \in \mathcal{S} \mid \hat{f}(s) \succcurlyeq x\})$ for all $x \in \mathcal{X}$.

${ }^{18}$ See also the earlier analysis of Segal $(1987,1990)$.
} 


$$
W_{S M}\left(\ldots ; x_{i} \text { on } E_{i} ; \ldots\right)=\int_{\pi \in \Pi} \phi\left(\sum_{i} U\left(x_{i}\right) \cdot \pi\left(E_{i}\right)\right) \cdot d \mu(\pi(\cdot))
$$

over purely subjective acts, and more generally

$$
\begin{aligned}
& W_{S M}\left(\ldots ;\left(\ldots ; x_{i j}, p_{i j} ; \ldots\right) \text { on } E_{i} ; \ldots\right) \\
& \quad=\int_{\pi \in \Pi} \phi\left(\sum_{i}\left[\sum_{j} U\left(x_{i j}\right) \cdot p_{i j}\right] \cdot \pi\left(E_{i}\right)\right) \cdot d \mu(\pi(\cdot))
\end{aligned}
$$

for some increasing cardinal functions $U(\cdot)$ and $\phi(\cdot)$, the family $\Pi$ of all subjective probability measures $\pi(\cdot)$ over events, and subjective probability measure $\mu(\cdot)$ over $\Pi$. For each $\pi(\cdot)$, the expected utility of the prospect $\left(\ldots ; x_{i}\right.$ on $\left.E_{i} ; \ldots\right)$ would be $\sum_{i} U\left(x_{i}\right) \cdot \pi\left(E_{i}\right)$, and the individual is averse to the uncertainty in these expected utility levels that results from their subjective uncertainty about $\pi(\cdot)$ as represented by $\mu(\cdot)$ (similarly for the mixed objective/subjective prospects in (10)). Risk aversion over objective uncertainty is captured by concavity of the utility function $U(\cdot)$, and ambiguity aversion captured by concavity of $\phi(\cdot)$.

The fourth major model of ambiguity aversion is the Variational Preferences Model of Maccheroni, Marinacci, and Rustichini (2006), which takes the form

$$
W_{V P}\left(\ldots ; x_{i} \text { on } E_{i} ; \ldots\right)=\min _{\pi \in \Pi}\left(\sum_{i} U\left(x_{i}\right) \cdot \pi\left(E_{i}\right)+\eta(\pi(\cdot))\right)
$$

over purely subjective acts, and more generally

$$
W_{V P}\left(\ldots ;\left(\ldots ; x_{i j}, p_{i j} ; \ldots\right) \text { on } E_{i} ; \ldots\right)=\min _{\pi \in \Pi}\left(\sum_{i}\left[\sum_{j} U\left(x_{i j}\right) \cdot p_{i j}\right] \cdot \pi\left(E_{i}\right)+\eta(\pi(\cdot))\right)
$$

for some increasing cardinal function $U(\cdot)$, the family $\Pi$ of all subjective probability measures $\pi(\cdot)$ over events, and nonnegative convex function $\eta(\cdot)$ over $\Pi$. For each prior $\pi(\cdot)$, the expected utility of the mixed objective/subjective bet $\left(\ldots ;\left(\ldots ; x_{i j}, p_{i j} ; \ldots\right)\right.$ on $\left.E_{i} ; \ldots\right)$, namely $\sum_{i}\left[\sum_{j} U\left(x_{i j}\right) \cdot p_{i j}\right] \cdot \pi\left(E_{i}\right)$, is supplemented by a value $\eta(\pi(\cdot))$ representing the individual's attitudes toward ambiguity, and the combined value then minimized over the family $\Pi$. The authors of this model have shown that this form includes the Multiple-Priors Model and the Multiplier Preferences Model of Hansen and Sargent (2001) as special cases.

It is important to note how these models are applied to Ellsberg-type bets. In order to fully separate and represent their objective and subjective uncertainty, such bets are expressed in the Anscombe-Aumann form $\left[\mathbf{P}_{1}\right.$ on $E_{1} ; \ldots ; \mathbf{P}_{n}$ on $\left.E_{n}\right]$, then evaluated as in equations (4), (6), (10), and (12). Thus, for Ellsberg's Three-Color Urn, the appropriate state space over which to apply the models is not the mixed objective/subjective space \{red, black, yellow\}, but rather the underlying purely subjective space $\left\{s_{0}, \ldots, s_{60}\right\}=\{0$ black balls, $\ldots, 60$ black balls $\}$, whose uncertainty is, 
after all, the underlying source of the urn's ambiguity. ${ }^{19}$ Expressed in this manner, Ellsberg's bets take the following form, where in each case $i$ runs from 0 to 60 :

$$
\begin{aligned}
& a_{1}=\left[\ldots ;\left(\$ 0, \frac{60}{90} ; \$ 100, \frac{30}{90}\right) \text { if } s_{i} ; \ldots\right] \\
& a_{2}=\left[\ldots ;\left(\$ 0, \frac{90-i}{90} ; \$ 100, \frac{i}{90}\right) \text { if } s_{i} ; \ldots\right] \\
& a_{3}=\left[\ldots ;\left(\$ 0, \frac{i}{90} ; \$ 100, \frac{90-i}{90}\right) \text { if } s_{i} ; \ldots\right] \\
& a_{4}=\left[\ldots ;\left(\$ 0, \frac{30}{90} ; \$ 100, \frac{60}{90}\right) \text { if } s_{i} ; \ldots\right] .
\end{aligned}
$$

Each of the above models has been shown to be consistent with standard Ellsberg-type preferences in the above and similar examples, each has been formally axiomatized by the above researchers, and as mentioned, each has seen applications in economics. 20

\section{Allais-Type Problems under Purely Subjective Uncertainty}

Our first observation is trivial: since the mixed-uncertainty forms (4), (6), (10), and (12) of the above models posit expected utility preferences over purely objective lotteries, they are directly subject to purely objective phenomena such as the Allais Paradox, Common Consequence Effect and Common Ratio Effect. ${ }^{21}$

Why did the developers of these models embed expected utility objective risk preferences into their forms? In the two-outcome world of the classic Ellsberg Paradoxes, the issue is of little consequence-if the only possible outcomes are $\{\$ 0, \$ 100\}$, it would be impossible to violate expected utility in choice over any menu of purely objective prospects.

Even if three or more possible outcomes are allowed, one could still argue that such models were not developed to address the Allais-type phenomenon of nonlinearity in probabilities, but rather the Ellsberg-type phenomenon of nonseparability across events, and it is only their ability to model the latter phenomenon which should matter. But it turns out that in a world of three or more outcomes and under natural regularity conditions, even the purely subjective Multiple-Priors, Smooth Ambiguity Preferences and Variational Preferences forms (3), (9), and (11) are subject to standard Allais-type difficulties.

The reason why Allais-type problems can extend to purely subjective uncertainty is that some purely subjective events can be said to be "more objective" than others. For the rest of this section, let $\mathcal{S}$ denote the normalized (e.g., thermometer) state space $\mathcal{S}=[0,1]$, partition it into $m$ equal-length intervals

\footnotetext{
${ }^{19}$ See the Appendix for a further discussion of this issue.

${ }^{20}$ See, e.g., Chamberlain (2001); Epstein (2001); Hansen and Sargent (2001); Mukerji and Tallon (2004); Klibanoff, Marinacci, and Mukerji (2005); Epstein and Schneider (2010); the papers in Gilboa (2004); and the 2011 Symposium Issue of Economic Theory in honor of the fiftieth anniversary of the Ellsberg Paradox (Ellsberg et al. 2011).

${ }^{21}$ See, e.g., Machina (1987).
} 
$\{[0,1 / m), \ldots,[i / m,(i+1) / m), \ldots,[(m-1) / m, 1]\}$, and for each $\alpha \in[0,1)$ define $[0, \alpha] \underset{m}{\times} \mathcal{S}$ as the union of the left $\alpha$ portions of these intervals, so that $[0, \alpha] \underset{m}{\times} \mathcal{S}=\cup_{i=0}^{m-1}[i / m,(i+\alpha) / m]$. As shown by Poincaré (1912) and others, ${ }^{22}$ such events will satisfy $\lim _{m \rightarrow \infty} \pi([0, \alpha] \underset{m}{\times} \mathcal{S})=\alpha$ for any measure $\pi(\cdot)$ over $\mathcal{S}$ with a sufficiently regular density.

More generally, for any set $\wp \subseteq[0,1)$ consisting of a finite union of intervals, and any positive integer $m$, define the purely subjective event $\wp \underset{m}{\times} \mathcal{S} \subseteq \mathcal{S}$ by

$$
\wp \underset{m}{\times} \mathcal{S}=\bigcup_{i=0}^{m-1}\{(i+\omega) / m \mid \omega \in \wp\} ;
$$

that is, as the union of the natural images of $\wp$ into each of $\mathcal{S}$ 's equal-length intervals. Events with this type of periodic structure are termed almost-objective events, and satisfy the convergence property $\lim _{m \rightarrow \infty} \pi(\wp \underset{m}{\times} \mathcal{S})=\lambda(\wp)$ for any subjective prior $\pi(\cdot)$ over $\mathcal{S}$ with a continuous (and therefore uniformly continuous) density function, where $\lambda(\cdot)$ is uniform Lebesgue measure over $[0,1]$. For any family of priors $\Pi_{0}$ whose family of densities is uniformly bounded and satisfies the uniform smoothness condition of uniform equicontinuity, ${ }^{23}$ such convergence will be uniform over $\Pi_{0}$ (we term this the uniform convergence property). Almost-objective events exhibit two important properties:

- Theoretically, event-smooth preference functions over purely subjective acts treat almost-objective events in much the same way as objective events: As $m \rightarrow \infty$ they exhibit exogenous, outcome-invariant revealed likelihoods of almost-objective events, independence of almost-objective and fixed subjective events, and probabilistically sophisticated betting preferences over almost-objective acts. ${ }^{24}$

- Empirically, while most so-called "objective" randomizing devices actually generate almost-objective uncertainty - the events \{heads, tails\} for a 50:50 coin are periodic in the (purely subjective) force of the flip, and each slot on a roulette wheel is periodic in the purely subjective force of the spin-bets on such events are viewed by both decision theorists and decision makers as purely objective lotteries. ${ }^{25}$

It is important to note that the convergence property $\lim _{m \rightarrow \infty} \pi(\wp \underset{m}{\times} \mathcal{S})=\lambda(\wp)$ implies the asymptotic equivalence of the purely subjective acts $\left[x_{1}\right.$ on $\wp_{1} \underset{m}{\times} \mathcal{S} ; \ldots ; x_{n}$ on $\left.\wp_{n} \underset{m}{\times} \mathcal{S}\right]$

\footnotetext{
${ }^{22}$ See Machina (2004, p. 9).

${ }^{23}$ Machina (2004, Thm.0). A family $\left\{\rho_{\tau}(\cdot) \mid \tau \in T\right\}$ of density functions over $\mathcal{S}$ is said to be uniformly equicontinuous if for any $\varepsilon>0$ there exists some $\delta>0$ such that $\left|s^{\prime}-s\right|<\delta$ implies $\left|\rho_{\tau}\left(s^{\prime}\right)-\rho_{\tau}(s)\right|<\varepsilon$ for all $s, s^{\prime} \in \mathcal{S}$ and $\tau \in T$. An example would be if $\left\{\rho_{\tau}(\cdot) \mid \tau \in T\right\}$ were the convex hull of a finite number of uniformly continuous densities. The phrase "... with uniformly bounded and uniformly continuous densities, ..." in the statement of Machina (2004, Thm. 0) should read “... with a uniformly bounded and uniformly equicontinuous family of densities,..." (It is this latter condition which is invoked in the proof of the theorem, and is necessary for our results).

${ }^{24}$ Machina (2004, Thms. 1, 2 \& 5, 3). The definition of event-smooth is that of Machina (2004, pp. 35-36; 2005, pp.16-17).

${ }^{25}$ Short of using quanta, how could one even construct a "purely objective" (as opposed to almost-objective) randomizing device?
} 
with their corresponding purely objective lotteries $\left(x_{1}, \lambda\left(\wp_{1}\right) ; \ldots ; x_{n}, \lambda\left(\wp_{n}\right)\right)$ regardless of the individual's actual prior $\pi(\cdot)$ over $\mathcal{S}$, so long as $\pi(\cdot)$ has a uniformly continuous density. For the analog thermometer of the Thermometer Problem, betting preferences on whether the thousandth (or millionth, ...) decimal of the temperature is even or odd will asymptote to betting preferences over objective 50:50 bets, regardless of whether one believes it is more likely to be hot than cold, or the other way around.

It would thus come as no surprise if, independently of agents' heterogeneous temperature beliefs, typical preferences over the purely subjective bets of the Thermometer Problem match typical preferences over their objective analoguesthat is, if they match the commonly observed Objective Common Ratio Effect rankings 26

$$
\begin{gathered}
\mathbf{P}_{1}=(\$ 6,000,0.45 ; \$ 0,0.55) \prec \mathbf{P}_{2}=(\$ 3,000,0.90 ; \$ 0,0.10) \\
\mathbf{P}_{3}=(\$ 6,000,0.001 ; \$ 0,0.999) \succ \mathbf{P}_{4}=(\$ 3,000,0.002 ; \$ 0,0.998)
\end{gathered}
$$

To generate a purely subjective version of this purely objective example, we posit some small enough $\varepsilon>0$ so that typical objective lottery preferences continue to satisfy

$$
\begin{gathered}
\hat{\mathbf{P}}_{1}=(\$ 6,000+\varepsilon, 0.45 ; \$ 0,0.55) \prec \mathbf{P}_{2}=(\$ 3,000,0.90 ; \$ 0,0.10) \\
\hat{\mathbf{P}}_{3}=(\$ 6,000-\varepsilon, 0.001 ; \$ 0,0.999) \succ \mathbf{P}_{4}=(\$ 3,000,0.002 ; \$ 0,0.998),
\end{gathered}
$$

in which case we would expect to observe the corresponding thermometer bet rankings

$$
\begin{gathered}
{[\$ 6 \mathrm{~K}+\varepsilon \text { on }[0,0.45] \underset{m}{\times} \mathcal{S} ; \$ 0 \text { on }(0.45,1] \underset{m}{\times} \mathcal{S}] \prec[\$ 3 \mathrm{~K} \text { on }[0,0.90] \underset{m}{\times} \mathcal{S} ; \$ 0 \text { on }(0.90,1] \underset{m}{\times} \mathcal{S}]} \\
{[\$ 6 \mathrm{~K}-\varepsilon \text { on }[0,0.001] \underset{m}{\times} \mathcal{S} ; \$ 0 \text { on }(0.001,1] \underset{m}{\times} \mathcal{S}] \succ[\$ 3 \mathrm{~K} \text { on }[0,0.002] \underset{m}{\times} \mathcal{S} ; \$ 0 \text { on }(0.002,1] \underset{m}{\times} \mathcal{S}]}
\end{gathered}
$$

for all large enough $m$, independently of a decision maker's priors over the temperature. ${ }^{27}$ Except for the small $\varepsilon$, these are the purely subjective Thermometer Problem bets at the beginning of the paper.

To see that these Subjective Common Ratio Effect preferences are inconsistent with the Subjective Expected Utility Model, observe that for any prior $\pi(\cdot)$ with a uniformly continuous density, the convergence property $\lim _{m \rightarrow \infty} \pi(\wp \underset{m}{\times} \mathcal{S})=\lambda(\wp)$ implies

\footnotetext{
${ }^{26}$ The earliest formal investigation of the Common Ratio Effect seems to be Ole Hagen's 1971 experiments as reported in Hagen (1973, 1979). MacCrimmon and Larsson (1979, pp. 350-59) provide a very thorough experimental treatment. The values used here are those of Kahneman and Tversky (1979, p. 267), who reported that 86 percent of their experimental subjects preferred $\mathbf{P}_{2}$ over $\mathbf{P}_{1}$, and 73 percent preferred $\mathbf{P}_{3}$ over $\mathbf{P}_{4}$.

${ }^{27}$ As with any such "effect," the hypothesized preferences (17) should be subjected to experimental testing and measurement.
} 


$$
\begin{aligned}
\lim _{m \rightarrow \infty} W_{S E U}\left(x_{1} \text { on } \wp_{1} \underset{m}{\times} \mathcal{S} ; \ldots ; x_{n} \text { on } \wp_{n} \underset{m}{\times} \mathcal{S}\right) & =\lim _{m \rightarrow \infty} \sum_{i=1}^{n} U\left(x_{i}\right) \cdot \pi\left(\wp_{i} \underset{m}{\times} \mathcal{S}\right) \\
& =\sum_{i=1}^{n} U\left(x_{i}\right) \cdot \lambda\left(\wp_{i}\right) .
\end{aligned}
$$

In other words, so long as $\pi(\cdot)$ has a uniformly continuous density, as $m \rightarrow \infty$ Subjective Expected Utility preferences will evaluate purely subjective acts of the form $\left[x_{1}\right.$ on $\wp_{1} \underset{m}{\times} \mathcal{S} ; \ldots ; x_{n}$ on $\left.\wp_{n} \underset{m}{\times} \mathcal{S}\right]$ according to the objective expected utility formula $\sum_{i=1}^{n} U\left(x_{i}\right) \cdot \lambda(\wp)$, independently of $\pi(\cdot)$. Setting $U(\$ 0)=0$, the rankings (17) imply the incompatible inequalities

$$
0.45 \cdot U(\$ 6 \mathrm{~K})
$$

$$
\begin{aligned}
& <0.45 \cdot U(\$ 6 \mathrm{~K}+\varepsilon)=\lim _{m \rightarrow \infty} W_{S E U}(\$ 6 \mathrm{~K}+\varepsilon \text { on }[0,0.45] \underset{m}{\times} \mathcal{S} ; \$ 0 \text { on }(0.45,1] \underset{m}{\times} \mathcal{S}) \\
& \leq \lim _{m \rightarrow \infty} W_{S E U}(\$ 3 \mathrm{~K} \text { on }[0,0.90] \underset{m}{\times} \mathcal{S} ; \$ 0 \text { on }(0.90,1] \underset{m}{\times} \mathcal{S})=0.90 \cdot U(\$ 3 \mathrm{~K})
\end{aligned}
$$

$0.001 \cdot U(\$ 6 \mathrm{~K})$

$$
\begin{aligned}
& >0.001 \cdot U(\$ 6 \mathrm{~K}-\varepsilon)=\lim _{m \rightarrow \infty} W_{S E U}(\$ 6 \mathrm{~K}-\varepsilon \text { on }[0,0.001] \underset{m}{\times} \mathcal{S} ; \$ 0 \text { on }(0.001,1] \underset{m}{\times} \mathcal{S}) \\
& \geq \lim _{m \rightarrow \infty} W_{S E U}(\$ 3 \mathrm{~K} \text { on }[0,0.002] \underset{m}{\times} \mathcal{S} ; \$ 0 \text { on }(0.002,1] \underset{m}{\times} \mathcal{S})=0.002 \cdot U(\$ 3 \mathrm{~K}),
\end{aligned}
$$

so that, just as the Objective Common Ratio Effect preferences (15) violate Objective Expected Utility, the Subjective Common Ratio Effect preferences (17) violate Subjective Expected Utility.

It should probably come as no surprise that Subjective Expected Utility preferences would be subject to a Subjective Common Ratio Effect. But is this just another example of an Ellsberg-type violation of the Sure-Thing Principle, which are already known to violate $S E U$ ? If so, we should expect that models of ambiguity aversion, which are designed precisely to handle Ellsberg, should not be subject to such difficulties.

In fact, the Subjective Common Ratio Effect is not an example of an Ellsberg-type violation of the Sure-Thing Principle or event-separability - in particular, even for fixed $m$ the pairs of bets in (17) are not related to each other in the Savage-like manner necessary to confirm or refute the Sure-Thing Principle. ${ }^{28}$ And while they can handle Ellsberg-type violations of event-separability, under standard regularity conditions most of the models we consider in this paper cannot handle the completely independent phenomenon of the Subjective Common Ratio Effect.

\footnotetext{
${ }^{28}$ Savage (1954, pp. $22-23$ ). The only common outcome value in the upper pair of bets is $\$ 0$, which cannot be replaced by any other common outcome value to yield the lower pair of bets. This holds even if the outcomes $\$ 6 \mathrm{~K}+\varepsilon$ and $\$ 6 \mathrm{~K}-\varepsilon$ in these bets are both replaced by $\$ 6 \mathrm{~K}$.
} 
To see this for the purely subjective Multiple-Priors form (3), take any bounded utility function $U(\cdot)$ and family of priors $\Pi_{0}$ with a uniformly bounded and uniformly equicontinuous family of densities over $\mathcal{S}$, and consider the model's limiting valuations of almost-objective acts $\left[x_{1}\right.$ on $\wp_{1} \underset{m}{\times} \mathcal{S} ; \ldots ; x_{n}$ on $\left.\wp_{n} \underset{m}{\times} \mathcal{S}\right]$. By the uniform convergence property, for each $\varepsilon>0$ there will exist some $m_{\varepsilon}$ such that

$$
\sum_{i=1}^{n} U\left(x_{i}\right) \cdot \lambda\left(\wp_{i}\right)-\varepsilon<\sum_{i=1}^{n} U\left(x_{i}\right) \cdot \pi\left(\wp_{i} \underset{m}{\times} \mathcal{S}\right)<\sum_{i=1}^{n} U\left(x_{i}\right) \cdot \lambda\left(\wp_{i}\right)+\varepsilon
$$

for all $\pi \in \Pi_{0}$ and all $m>m_{\varepsilon}$. This in turn implies

$$
\sum_{i=1}^{n} U\left(x_{i}\right) \cdot \lambda\left(\wp_{i}\right)-\varepsilon \leq \min _{\pi \in \Pi_{0}} \sum_{i=1}^{n} U\left(x_{i}\right) \cdot \pi\left(\wp_{i} \underset{m}{\times} \mathcal{S}\right)<\sum_{i=1}^{n} U\left(x_{i}\right) \cdot \lambda\left(\wp_{i}\right)+\varepsilon
$$

for all $m>m_{\varepsilon}$, and thus that

$$
\text { (22) } \begin{aligned}
\lim _{m \rightarrow \infty} W_{M P}\left(x_{1} \text { on } \wp_{1} \underset{m}{\times} \mathcal{S} ; \ldots ; x_{n} \text { on } \wp_{n} \underset{m}{\times} \mathcal{S}\right)=\lim _{m \rightarrow \infty} \min _{\pi \in \Pi_{0}} \sum_{i=1}^{n} U\left(x_{i}\right) \cdot \pi\left(\wp_{i} \underset{m}{\times} \mathcal{S}\right) \\
=\min _{\pi \in \Pi_{0}} \lim _{m \rightarrow \infty} \sum_{i=1}^{n} U\left(x_{i}\right) \cdot \pi\left(\wp_{i} \underset{m}{\times} \mathcal{S}\right)=\min _{\pi \in \Pi_{0}} \sum_{i=1}^{n} U\left(x_{i}\right) \cdot \lambda\left(\wp_{i}\right)=\sum_{i=1}^{n} U\left(x_{i}\right) \cdot \lambda\left(\wp_{i}\right) .
\end{aligned}
$$

Thus, as with Subjective Expected Utility preferences, as $m \rightarrow \infty$ Multiple-Priors preferences with a sufficiently regular $\Pi_{0}$ evaluate purely subjective acts of the form $\left[x_{1}\right.$ on $\wp_{1} \underset{m}{\times} \mathcal{S} ; \ldots ; x_{n}$ on $\left.\wp_{n} \underset{m}{\times} \mathcal{S}\right]$ according to the objective expected utility formula $\sum_{i=1}^{n} U\left(x_{i}\right) \cdot \lambda\left(\wp_{i}\right)$, and are accordingly subject to the Subjective Common Ratio Effect in precisely the same manner as Subjective Expected Utility preferences.

To see this for the purely subjective Smooth Ambiguity Preferences form (9), assume $\phi(\cdot)$ is uniformly continuous and that the closure of $\mu(\cdot)$ is a uniformly bounded and uniformly equicontinuous family of density functions, which together imply

$$
\begin{aligned}
\lim _{m \rightarrow \infty} & W_{S M}\left(x_{1} \text { on } \wp_{1} \underset{m}{\times} \mathcal{S} ; \ldots ; x_{n} \text { on } \wp_{n} \underset{m}{\times} \mathcal{S}\right) \\
= & \lim _{m \rightarrow \infty} \int_{\pi \in \Pi} \phi\left(\sum_{i=1}^{n} U\left(x_{i}\right) \cdot \pi\left(\wp_{i} \underset{m}{\times} \mathcal{S}\right)\right) \cdot d \mu(\pi(\cdot)) \\
= & \int_{\pi \in \Pi} \phi\left(\lim _{m \rightarrow \infty} \sum_{i=1}^{n} U\left(x_{i}\right) \cdot \pi\left(\wp_{i} \underset{m}{\times} \mathcal{S}\right)\right) \cdot d \mu(\pi(\cdot)) \\
= & \int_{\pi \in \Pi} \phi\left(\sum_{i=1}^{n} U\left(x_{i}\right) \cdot \lambda\left(\wp_{i}\right)\right) \cdot d \mu(\pi(\cdot))=\phi\left(\sum_{i=1}^{n} U\left(x_{i}\right) \cdot \lambda\left(\wp_{i}\right)\right) .
\end{aligned}
$$


Thus, as with the Subjective Expected Utility and Multiple-Priors Models, as $m \rightarrow \infty$ the Smooth Ambiguity Preferences Model evaluates purely subjective acts of the form $\left[x_{1}\right.$ on $\wp_{1} \underset{m}{\times} \mathcal{S} ; \ldots ; x_{n}$ on $\left.\wp_{n} \times \underset{m}{\times} \mathcal{S}\right]$ according to (an ordinal transformation of) the formula $\sum_{i=1}^{n} U\left(x_{i}\right) \cdot \lambda\left(\wp_{i}\right),{ }^{29}$ and is therefore similarly subject to the Subjective Common Ratio Effect.

Such preferences are also incompatible with the purely subjective Variational Preferences form (11). Provided the domain $\Pi_{0}$ over which $\eta(\cdot)<+\infty$ consists of a uniformly bounded and uniformly equicontinuous family of densities, and $\eta(\cdot)$ is uniformly continuous over $\Pi_{0}$, we have

$$
\begin{aligned}
\lim _{m \rightarrow \infty} & W_{V P}\left(x_{1} \text { on } \wp_{1} \underset{m}{\times} \mathcal{S} ; \ldots ; x_{n} \text { on } \wp_{n} \underset{m}{\times} \mathcal{S}\right) \\
= & \lim _{m \rightarrow \infty} \min _{\pi \in \Pi_{0}}\left(\sum_{i=1}^{n} U\left(x_{i}\right) \cdot \pi\left(\wp_{i} \underset{m}{\times} \mathcal{S}\right)+\eta(\pi(\cdot))\right) \\
= & \min _{\pi \in \Pi_{0}}\left(\lim _{m \rightarrow \infty} \sum_{i=1}^{n} U\left(x_{i}\right) \cdot \pi\left(\wp_{i} \underset{m}{\times} \mathcal{S}\right)+\eta(\pi(\cdot))\right) \\
= & \min _{\pi \in \Pi_{0}}\left(\sum_{i=1}^{n} U\left(x_{i}\right) \cdot \lambda\left(\wp_{i}\right)+\eta(\pi(\cdot))\right) \\
= & \sum_{i=1}^{n} U\left(x_{i}\right) \cdot \lambda\left(\wp_{i}\right)+\min _{\pi \in \Pi_{0}} \eta(\pi(\cdot)) .
\end{aligned}
$$

Since the second term in the final expression takes the same value for all prospects $\left[x_{1}\right.$ on $\wp_{1} \underset{m}{\times} \mathrm{S} ; \ldots ; x_{n}$ on $\left.\wp_{n} \underset{m}{\times} \mathcal{S}\right]$, preferences over such prospects are again ordinally equivalent to the formula $\sum_{i=1}^{n} U\left(x_{i}\right) \cdot \lambda\left(\wp_{i}\right)$, and are therefore similarly subject to the Subjective Common Ratio Effect.

Again, it is important to note that this Allais-type difficulty is not due to the conditional objective expected utility property (the bracketed terms) of these model's mixed-uncertainty forms (4), (10), and (12). It is a feature of how they model attitudes toward purely subjective uncertainty - that is, a feature of their purely subjective forms (3), (9), and (11).

The two important special cases of the Rank-Dependent form $W_{R D}(\cdot)$ have opposite properties in this regard. As noted, the convex-capacity form $W_{R D \text { convex }}(\cdot)$ (form (7)) can be expressed as a Multiple-Priors form (3) for some family of priors $\Pi_{0}$. Thus, whenever $\Pi_{0}$ consists of a uniformly bounded and uniformly equicontinuous family of densities, the argument of equations (20)-(22) establishes that $W_{R D \text { convex }}(\cdot)$ is violated by the Subjective Common Ratio Effect preferences (17).

The Subjective Common Ratio preferences (17) are, however, compatible with the other important case special of the Rank-Dependent Model, namely the $W_{\text {RDdistortion }}(\cdot)$ form $(8)$. When the base measure $\pi(\cdot)$ has a continuous (and therefore uniformly continuous) density over $\mathcal{S}$, the uniform convergence property implies 
(25)

$$
\begin{aligned}
\lim _{m \rightarrow \infty} & W_{R \text { distortion }}\left(x_{1} \text { on } \wp_{1} \underset{m}{\times} \mathcal{S} ; \ldots ; x_{n} \text { on } \wp_{n} \underset{m}{\times} \mathcal{S}\right) \\
& =\lim _{m \rightarrow \infty} \sum_{i=1}^{n} U\left(x_{i}\right) \cdot\left[G\left(\sum_{k=1}^{i} \pi\left(\wp_{k} \underset{m}{\times} \mathcal{S}\right)\right)-G\left(\sum_{k=1}^{i-1} \pi\left(\wp_{k} \underset{m}{\times} \mathcal{S}\right)\right)\right] \\
& =\sum_{i=1}^{n} U\left(x_{i}\right) \cdot\left[G\left(\sum_{k=1}^{i} \lim _{m \rightarrow \infty} \pi\left(\wp_{k} \underset{m}{\times} \mathcal{S}\right)\right)-G\left(\sum_{k=1}^{i-1} \lim _{m \rightarrow \infty} \pi\left(\wp_{k} \underset{m}{\times} \mathcal{S}\right)\right)\right] \\
& =\sum_{i=1}^{n} U\left(x_{i}\right) \cdot\left[G\left(\sum_{k=1}^{i} \lambda\left(\wp_{k}\right)\right)-G\left(\sum_{k=1}^{i-1} \lambda\left(\wp_{k}\right)\right)\right] .
\end{aligned}
$$

In other words, so long as $\pi(\cdot)$ has a uniformly continuous density, $W_{R D \text { distortion }}(\cdot)$ preferences over almost-objective acts $\left[x_{1}\right.$ on $\wp_{1} \underset{m}{\times} \mathcal{S} ; \ldots ; x_{n}$ on $\left.\wp_{n} \underset{m}{\times} \mathcal{S}\right]$ converge to Quiggin-type Anticipated Utility preferences of the form $\sum_{i=1}^{n} U\left(x_{i}\right) \cdot\left[G\left(\sum_{k=1}^{i} p_{k}\right)-\right.$ $\left.G\left(\sum_{k=1}^{i=1} p_{k}\right)\right]$ over their corresponding purely objective lotteries, which for appropriate choice of $G(\cdot)$ has been shown to indeed be compatible with Common Ratio Effect preferences (Quiggin 1982; Tversky and Kahneman 1992).30

\section{Attitudes toward Different Sources and/or Amounts of Ambiguity}

Although the Rank-Dependent Model has at least one special case (8) which is not subject to the Allais-type problems of the previous section, its general form (5) is subject to a different type of difficulty.

In bets that involve only two outcomes, such as the classic Ellsberg examples, the outcome values are necessarily adjacent to each other. However, adding a third possible outcome to an Ellsberg-type bet allows for possible interactions between outcome values which are not necessarily adjacent, such as the outcomes $+\$ 8,000$ and $-\$ 8,000$ in the Slightly-Bent Coin Problem.

The Slightly-Bent Coin Problem differs from the examples of the previous section and from most other Allais- and Ellsberg-type examples in that it only involves a single pair of prospects. In the author's view, the ambiguity-averse choice would be for Bet I, which spreads the uncertainty of receiving $+\$ 8,000$ versus $-\$ 8,000$ across the less ambiguous coin rather than the more ambiguous urn. Others have told me they would prefer Bet II. A strict preference in either direction would violate the Subjective Expected Utility Model: since informational symmetry would imply $\pi(B H)$ (i.e., $\pi($ black, heads $))=\pi(B T)=\pi(W H)=\pi(W T)=1 / 4$, both bets would have a subjective expected utility of $\frac{1}{4} \cdot U(+\$ 8,000)+\frac{1}{2} \cdot U(\$ 0)+\frac{1}{4} \cdot U(-\$ 8,000)$.

\footnotetext{
${ }^{30}$ What about the intersection case of $W_{R D d i s t o r t i o n}(\cdot)$ with a convex $G(\cdot)$, which is also a special case of $W_{M P}(\cdot)$ ? Why does the argument of (25) hold in this case rather than (22)? The reason is that while a preference function $W_{R D \text { distortion }}(\cdot)$ with a convex $G(\cdot)$ can indeed be represented in the multiple-priors form (3), such a representation must involve a family $\Pi_{0}$ which is necessarily not uniformly equicontinuous (Carlier and Dana 2003), and for which the second equality in (22) necessarily fails. Uniform convergence is not an issue for the argument of (25) as it involves only a single prior, namely the base measure $\pi(\cdot)$ of $(8)$, and whose third inequality holds so long as $\pi(\cdot)$ has a (uniformly) continuous density.
} 
Although the ambiguity-averse ranking Bet I $\succ$ Bet II is consistent with the Multiple-Priors, Smooth Ambiguity Preferences, and Variational Preferences Models, neither it nor the reverse ranking Bet I $\prec$ Bet II is consistent with the Rank-Dependent Model, which implies that the two bets must be indifferent to each other. Although their good-news events $B H$ and $W T$ for the payoff $+\$ 8,000$ are not the same, they are informationally symmetric, which would presumably imply $C(B H)=C(W T)$. The two bets $d o$ have the same good-news event for the payoff $\$ 0$, namely $B H \cup W H \cup W T$. Together, this implies that the values

$$
\begin{aligned}
(26) W_{R D}(\mathrm{BET} \mathrm{I})= & U(+\$ 8,000) \cdot C(B H)+U(\$ 0) \cdot[C(B H \cup W H \cup W T)-C(B H)] \\
& +U(-\$ 8,000) \cdot[1-C(B H \cup W H \cup W T)] \\
W_{R D}(\text { BETII })= & U(+\$ 8,000) \cdot C(W T)+U(\$ 0) \cdot[C(B H \cup W H \cup W T)-C(W T)] \\
& +U(-\$ 8,000) \cdot[1-C(B H \cup W H \cup W T)]
\end{aligned}
$$

must be equal, so that the Rank-Dependent Model implies indifference between these two purely subjective bets. In other words, the Rank-Dependent Model cannot represent a strict preference, in either direction, for one of these sources or amounts of ambiguity (coin versus urn) over the other. This is presumably relevant if realworld decisions involve different sources, with different amounts, of ambiguity. To see that the difficulty arises from the existence of a third outcome value in these bets, recall that ambiguity aversion over their two-outcome analogues $(+\$ 8,000$ if heads; $-\$ 8,000$ if tails) and ( $+\$ 8,000$ if white; $-\$ 8,000$ if black) can be easily modeled by assuming $C(B H \cup W H)>C(W H \cup W T) .{ }^{31}$

In fact, the difficulties raised by the two-source Slightly-Bent Coin Problem do not require separate sources of uncertainty, but can arise with bets over a single source of subjective uncertainty, when different bets on it have different amounts of ambiguity. Say you've just learned that a meteor of unknown size or speed has been spotted, is expected to strike the earth sometime in the next 13 hours, and you are betting on a single subjective variable, namely the longitude $\ell$ of its strike. Since you know nothing more, from your point of view the circular state space $\left[0^{\circ}, 360^{\circ}\right)$ is informationally symmetric, but since there's subjective uncertainty, your capacity $C(\cdot)$ may still be nonadditive. The following bets are based on whether the meteor hits the earth in the Western or Eastern Hemisphere, and whether the seventh decimal of $\ell$ is even or odd. This is a single-source analogue of the Slightly-Bent Coin Problem, where the highly subjective urn is replaced by the highly subjective partition $\left\{\left[0^{\circ}, 180^{\circ}\right),\left[180^{\circ}, 360^{\circ}\right)\right\}$, and the slightly-bent coin is replaced by the almost-objective partition \{seventh decimal odd, seventh decimal even\}. ${ }^{32}$

\footnotetext{
${ }^{31}$ Why use a bent coin in the original example? Why not use an objective 50:50 coin to make the same point? As noted by Lo (2008, footnote 1), such an example does appear in a manuscript version of Machina (2009). (Lo applies this version to the model of Klibanoff 2001, and Blavatskyy 2013 applies it to other models.) We use a bent coin here to show that this three-outcome difficulty for the Rank-Dependent Model extends from mixed bets involving both objective and subjective sources of uncertainty to purely subjective bets involving different sources and degrees of ambiguity.

${ }^{32}$ Since it is based on a single source of purely subjective uncertainty, this example also differs from the Reflection Example and 50:51 Example of Machina (2009), each of which involves both objective and subjective uncertainty.
} 
Meteorite Problem $(\ell=$ Longitude of Strike $)$

\begin{tabular}{rrrrrrr}
\hline \hline & \multicolumn{2}{c}{ Bet I } & & \multicolumn{2}{c}{ Bet II } \\
\cline { 2 - 3 } \cline { 5 - 6 } & & & $\ell \in\left[0^{\circ}, 180^{\circ}\right)$ & $\ell \in\left[180^{\circ}, 360^{\circ}\right)$ \\
\hline 7th decimal even & $+\$ 8,000$ & $\$ 0$ & versus & 7th decimal even & $\$ 0$ & $\$ 0$ \\
7th decimal odd & $-\$ 8,000$ & $\$ 0$ & & 7th decimal odd & $-\$ 8,000$ & $+\$ 8,000$ \\
\hline
\end{tabular}

Presumably, an ambiguity-averse decision maker would prefer that the $\pm \$ 8,000$ uncertainty depend upon the seventh decimal than on the hemisphere, and prefer Bet I over Bet II, and it is reasonable to say that Bet I is less ambiguous than Bet II. But since the good-news events for the payoff $\$ 8,000$ are again informationally symmetric, and the good-news events for $\$ 0$ are again identical, the Rank-Dependent Model cannot allow a strict preference for (or against) the less ambiguous bet.

\section{Attitudes toward Ambiguity at Different Outcome Levels}

A third aspect of ambiguity aversion which can arise in a world of three or more outcomes is that, as with objective risk, ambiguity can occur at or across different final wealth levels or different gain/loss levels in a prospect, and individuals may exhibit different attitudes toward ambiguity at these different outcome levels. This cannot be seen in the three-outcome bets of the Slightly-Bent Coin or Meteorite Problems, where in each bet, the upper and lower outcomes $+\$ 8,000$ and $-\$ 8,000$ enter with equal ambiguity. But in general, prospects with three or more outcomes can exhibit more ambiguity at some outcome levels than others, which can allow this third aspect of ambiguity aversion to reveal itself.

This can be seen from the three-color Ambiguity at Low versus High Outcomes Problem at the beginning of the paper. Recall that each urn's bet involves the same triple of outcomes $\$ 0, \$ c$, and $\$ 100$, where $\$ c$ is the decision maker's (or your own) certainty equivalent of the objective lottery $\left(\$ 0, \frac{1}{2} ; \$ 100, \frac{1}{2}\right) \cdot 33$ But as noted, the ambiguity in Urn I is across the lower- and middle-outcome values $\$ 0$ and $\$ c$, whereas in Urn II it is across the middle- and upper-outcome values $\$ c$ and $\$ 100$. If ambiguity aversion somehow involves pessimism, might not an ambiguity averter have a strict preference for Urn II over Urn I, just as a risk averter might prefer bearing risk about higher rather than lower outcome levels?

Maybe yes, maybe no. But whether or not an ambiguity averter should prefer one bet over the other, none of the models we are considering allow this to happen in either direction.

Unlike the examples of the previous two sections, but like the classic Ellsberg examples, this and the other examples of this section involve mixed objective/ subjective uncertainty. Accordingly, we express their bets in a manner which separates their objective from their subjective uncertainty-that is, as AnscombeAumann acts—-so they can be evaluated by the models' formulas (4), (6), (10), and

\footnotetext{
${ }^{33}$ Lest the value $\$ c$ be interpreted as coming from backward induction of some two-stage prospects, we assume that this monetary amount has been independently and previously elicited from preferences over single-stage, purely objective lotteries. Similarly for the monetary values yielding the exogenously specified utility levels in the other examples of this section, as well as the probability $\hat{p}$ for which $G(\hat{p})=1 / 2$.
} 
Ambiguity at Low versus High Outcomes Problem-Three-Color Version: Mapping from States to ObJective LotTeries and ExPECted Utility Values

\begin{tabular}{lcccc}
\hline \hline & $B B$ & $B W$ & $W B$ & $W W$ \\
\hline Urn I & $\left(\$ 0, \frac{2}{3} ; \$ 100, \frac{1}{3}\right)$ & $\left(\$ 0, \frac{1}{3} ; \$ c, \frac{1}{3} ; \$ 100, \frac{1}{3}\right)$ & $\left(\$ 0, \frac{1}{3} ; \$ c, \frac{1}{3} ; \$ 100, \frac{1}{3}\right)$ & $\left(\$ c, \frac{2}{3} ; \$ 100, \frac{1}{3}\right)$ \\
Urn II & $\left(\$ 0, \frac{1}{3} ; \$ c, \frac{2}{3}\right)$ & $\left(\$ 0, \frac{1}{3} ; \$ c, \frac{1}{3} ; \$ 100, \frac{1}{3}\right)$ & $\left(\$ 0, \frac{1}{3} ; \$ c, \frac{1}{3} ; \$ 100, \frac{1}{3}\right)$ & $\left(\$ 0, \frac{1}{3} ; \$ 100, \frac{2}{3}\right)$ \\
Expected utility & $\frac{1}{3}$ & $\frac{1}{2}$ & $\frac{1}{2}$ & $\frac{2}{3}$ \\
\hline
\end{tabular}

(12). The above table presents these mappings from states to objective lotteries, where the underlying subjective uncertainty of each urn is given by its state space $\{B B, B W, W B, W W\}$ denoting the respective colors of its two unknown-color balls. For example, when both the unknown-color balls in Urn I are black, it yields a $2 / 3$ chance of paying $\$ 0$ and a $1 / 3$ chance of paying $\$ 100$; if both are white, it yields a $2 / 3$ chance of $\$ c$ and a $1 / 3$ chance of $\$ 100$, etc. Under the conditional objective expected utility hypothesis adopted by each of the four models (the bracketed terms in equations (4), (6), (10) and (12)), we can assign utility values of $\{0,1 / 2,1\}$ to the outcomes $\{\$ 0, \$ c, \$ 100\}$ to obtain and equations (4), (6), (10), and (12) imply

Multiple-Priors Model (4):

$$
\begin{aligned}
W_{M P}(\mathrm{Urn} \mathrm{I}) & =\min _{\substack{\left(p_{B B}, p_{B W}, p_{W B}, p_{W W}\right) \\
\in \Pi_{0}}}\left[\frac{1}{3} \cdot p_{B B}+\frac{1}{2} \cdot p_{B W}+\frac{1}{2} \cdot p_{W B}+\frac{2}{3} \cdot p_{W W}\right] \\
& =W_{M P}(\mathrm{Urn} \mathrm{II}) .
\end{aligned}
$$

Rank-Dependent Model (6):

$$
\begin{aligned}
W_{R D}(\mathrm{Urn} \mathrm{I})= & \frac{2}{3} \cdot C(W W)+\frac{1}{2} \cdot[C(W W \cup B W \cup W B)-C(W W)] \\
& +\frac{1}{3} \cdot[1-C(W W \cup B W \cup W B)]=W_{R D}(\mathrm{Urn} \mathrm{II}) .
\end{aligned}
$$

Smooth Ambiguity Preferences Model (10):

$$
\begin{aligned}
& W_{S M}(\mathrm{Urn} \mathrm{I}) \\
& =\int \phi\left(\frac{1}{3} \cdot p_{B B}+\frac{1}{2} \cdot p_{B W}+\frac{1}{2} \cdot p_{W B}+\frac{2}{3} \cdot p_{W W}\right) \cdot d \mu\left(p_{B B}, p_{B W}, p_{W B}, p_{W W}\right) \\
& =W_{S M}(\mathrm{Urn} \mathrm{II}) .
\end{aligned}
$$

Variational Preferences Model (12):

$$
\begin{aligned}
& \text { (30) } W_{V P}(\mathrm{Urn} \mathrm{I}) \\
& =\min _{\substack{\left(p_{B B}, p_{B W}, P_{W B}, p_{W W}\right) \\
\in \Pi}}\left[\frac{1}{3} \cdot p_{B B}+\frac{1}{2} \cdot p_{B W}+\frac{1}{2} \cdot p_{W B}+\frac{2}{3} \cdot p_{W W}+\eta\left(p_{B B}, p_{B W}, p_{W B}, p_{W W}\right)\right] \\
& =W_{V P}(\mathrm{Urn} \text { II }) .
\end{aligned}
$$


In other words, none of the four models allow the decision maker to exhibit an aversion to ambiguity in the lower outcome levels relative to ambiguity in the upper outcome levels or vice versa, regardless of their subjective beliefs as represented by $\Pi_{0}, C(\cdot), \mu(\cdot)$ or $\eta(\cdot)$.

What is it in the structure of these bets that necessitates this indifference? As seen from the first two rows of the above table, the two bets have different mappings from states to objective lotteries. But as seen in the third row, they have the same statewise assignment of conditional expected utility values. Any decision model that evaluates a mixed-uncertainty bet solely on its mapping from states to these values-as do the Multiple-Priors, Rank-Dependent, Smooth Ambiguity Preferences, and Variational Preferences Models ${ }^{34}$ - must necessarily be indifferent between the two bets, in spite of the fact that their ambiguity occurs at different outcome levels. ${ }^{35}$

One might argue that preferences for Urn I versus Urn II in this example would not be a feature of an individual's ambiguity preferences at all, but rather, determined by their attitudes toward objective risk. But objective risk attitudes are completely incorporated into the value of $\$ c$ and hence into the statewise conditional expected utility levels. Preferences over these bets can only reflect attitudes toward ambiguity at high versus low outcome levels_-attitudes which cannot be captured by either Subjective Expected Utility or any of the major models.

The use of four-outcome bets in Example 1 below allows for a more explicit illustration of this phenomenon. Again, subsume objective risk attitudes by selecting monetary outcomes with equally spaced utility levels 1 through 4 , and consider the following bets expressed in terms of these utility levels. ${ }^{36}$ Urn I is obtained from the purely objective bet $\hat{b}_{0}$ by introducing ambiguity across its lower outcome values 1 and 2 , whereas Urn II is obtained from $\hat{b}_{0}$ by introducing ambiguity across its higher outcome values 3 and 4 .

Informational symmetry implies that a subjective expected utility maximizer would have equal subjective probabilities over the states $\{B B, B W, W B, W W\}$, and accordingly be indifferent between the bets. But if ambiguity in lower outcome levels matters differently than ambiguity in higher outcome levels, an ambiguity averter may well have a strict preference for one bet over the other. But since the two urns again have the same mappings from states to expected utility levels, the four models must again rank them as indifferent—none can allow for a preference (or aversion) toward ambiguity about some outcome values rather than others.

Example 2 below differs from the previous one in that, rather than introducing ambiguity across different outcome levels, ambiguity is generated by changes in the outcome levels themselves. Starting from purely objective 50:50 urns yielding outcomes $\{2,4\}$, ambiguity is introduced into Urn I via an informationally symmetric spread about its lower value 2, and into Urn II via an informationally

\footnotetext{
${ }^{34}$ This follows since the conditional objective lotteries $\left(\ldots ; x_{i j}, p_{i j} ; \ldots\right)$ enter only through the bracket terms $\left[\sum_{j} U\left(x_{i j}\right) \cdot p_{i j}\right]$ in equations $(4),(6),(10)$, and (12).

${ }^{35}$ One might argue that the two bets do not have the same statewise assignments from states to expected utility levels, since their state spaces $\{B B$ in Urn I, $B W$ in Urn I, . . $\}$ and $\{B B$ in Urn II, $B W$ in Urn II, ... $\}$ are distinct. The use of separate urns in this example is for clarity of presentation-replacing Urn II's bet by the bet $\{\$ c$ if black; $\$ 100$ if white; $\$ 0$ if red $\}$ on Urn I yields an equivalent example over the common state space $\{B B$ in Urn I, $B W$ in Urn I, $W B$ in Urn I, $W W$ in Urn I $\}$. A similar argument holds for the following examples.

${ }^{36}$ As in footnote 33 , these monetary values are assumed to have been independently elicited.
} 
Ambiguity at Low versus High Outcomes Problem-Four-Color Version, Example 1

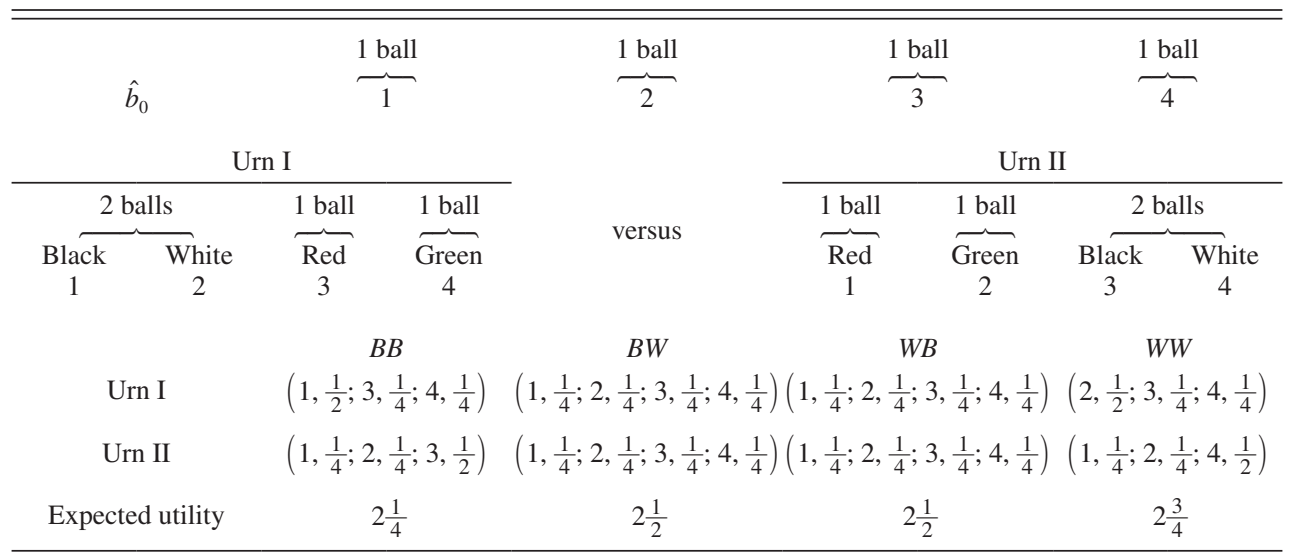

Ambiguity at Low versus High Outcomes Problem-Four-Color Version, Example 2

\begin{tabular}{|c|c|c|c|c|c|c|c|c|}
\hline \multicolumn{4}{|c|}{ Urn I } & & \multicolumn{4}{|c|}{ Urn II } \\
\hline \multicolumn{2}{|c|}{1 ball } & \multicolumn{2}{|c|}{1 ball } & & \multicolumn{2}{|c|}{1 ball } & \multicolumn{2}{|c|}{1 ball } \\
\hline Black & White & Red & $\overline{\text { Green }}$ & & Red & Green & Black & White \\
\hline 1 & 3 & 4 & 4 & versus & 2 & 2 & 3 & 5 \\
\hline
\end{tabular}

symmetric spread about its upper value 4 . Since each urn has the same mapping $\{B R, B G, W R, W G\} \rightarrow\left\{2 \frac{1}{2}, 2 \frac{1}{2}, 3 \frac{1}{2}, 3 \frac{1}{2}\right\}$ from states to expected utility levels, the four models must again rank the bets as indifferent.

One might think that the problem posed by these examples-namely that their different bets imply the same mappings from states to expected utility levels-could be averted by simply replacing the conditional objective expected utility terms $\sum_{j} U\left(x_{i j}\right) \cdot p_{i j}$ in formulas (4), (6), (10), and (12) with a Quiggin-type Anticipated Utility term $\sum_{j} U\left(x_{i j}\right) \cdot\left[G\left(\sum_{k=1}^{j} p_{i k}\right)-G\left(\sum_{k=1}^{j-1} p_{i k}\right)\right]$ with appropriate choice of cumulative probability transformation function $G(\cdot) \cdot{ }^{37}$ However, such a replacement doesn't help.

To see this, subsume attitudes toward objective risk by selecting $\hat{p}$ for which $G(\hat{p})=1 / 2$, and consider the following bets, each based on the flip of an objective $\hat{p}:(1-\hat{p})$ coin and a single-ball urn. Similar to the previous example, Bet I differs from the objective lottery $(4, \hat{p} ; 2,1-\hat{p})$ by introducing informationally symmetric ambiguity about the lower value 2 , whereas Bet II differs from it by introducing ambiguity about the higher value 4 . The bets again have the same mapping \{black, white $\} \rightarrow\left\{2 \frac{1}{2}, 3 \frac{1}{2}\right\}$ from subjective states to conditional anticipated utility levels, so models which evaluate prospects solely on the basis of these mappings must again rank the bets as indifferent, and again cannot exhibit a preference for ambiguity in high versus low outcome values.

\footnotetext{
${ }^{37}$ See, e.g., Klibanoff, Marinacci, and Mukerji (2005, pp. 1859-60). Outcome values and events in the RankDependent case should be labeled so that the terms $\sum_{j} U\left(x_{i j}\right) \cdot\left[G\left(\sum_{k=1}^{j} p_{i k}\right)-G\left(\sum_{k=1}^{j-1} p_{i k}\right)\right]$ are nonincreasing in $i$, and for each $i$ the values $x_{i j}$ are nonincreasing in $j$.
} 
Ambiguity at Low versus High Outcomes-Anticipated Utility Version $\operatorname{prob}($ heads:tails $)=\hat{p}: 1-\hat{p} \quad G(\hat{p})=\frac{1}{2}$

\begin{tabular}{|c|c|c|c|c|c|c|}
\hline & \multicolumn{2}{|c|}{ Bet I } & & & \multicolumn{2}{|c|}{ Bet II } \\
\hline & \multicolumn{2}{|c|}{1 ball } & & & \multicolumn{2}{|c|}{1 ball } \\
\hline & Black & White & & & Black & White \\
\hline Heads & 4 & 4 & & Heads & 3 & 5 \\
\hline Tails & 1 & 3 & versus & Tails & 2 & 2 \\
\hline
\end{tabular}

It is worth contrasting a preference for ambiguity at high rather than low outcome values with what might be called decreasing absolute ambiguity aversion. ${ }^{38}$ This distinction can be illustrated by analogy with the case of objective risk preferences. Decreasing absolute risk aversion in the standard objective sense involves starting with a pair of prospects, adding some common $\Delta x$ to all their monetary outcomes, and comparing the ranking over the newly created pair with the ranking over the original pair. In contrast, a preference for risk about higher rather than lower outcomes starts with a single prospect, and compares whether the individual would prefer a given mean-preserving spread about a higher rather than a lower outcome value, without changing the values of other outcomes. ${ }^{39}$ These properties of preferences are not equivalent even under objective expected utility: under expected utility, the first is equivalent to $-U^{\prime \prime}(x) / U^{\prime}(x)$ decreasing in $x$, whereas the second is equivalent to the weaker condition that $-U^{\prime \prime}(x)$ is decreasing in $x$, and includes the case of constant absolute risk aversion.

The distinction in the case of ambiguity preferences is analogous. Decreasing absolute ambiguity aversion would presumably involve adding some $\Delta U$ to all the utility outcomes of a pair of prospects, and comparing the ranking over the second pair with the ranking over the first. Although decreasing absolute ambiguity aversion cannot be exhibited by the Multiple-Priors, Rank-Dependent, or Variational Preferences forms (4), (6), or (12), ${ }^{40}$ they can be easily adapted by putting their conditional expected utility terms $\sum_{j} U\left(x_{i j}\right) \cdot p_{i j}$ inside an appropriate nonlinear function (or in the case of the Smooth Ambiguity Preferences Model (10), by simply making the appropriate curvature assumptions on $\phi(\cdot))$. On the other hand, a preference for ambiguity about higher rather than lower outcomes starts with a single purely objective prospect, and compares whether the individual would prefer introducing ambiguity in higher rather than lower utility levels, holding the other values constant. Since the examples of this section involve pairs of prospects with identical mappings from states to conditional expected utility levels, they imply that none of the four models can exhibit a preference for ambiguity about higher rather than lower outcome values (or vice versa), regardless of the shape of their $U(\cdot)$ functions, or whether their conditional expected utility terms are nonlinearly transformed.

Our final example, following up on the discussion of Ellsberg (2001, pp. 199-200), is that of a consumer, who by paying 1 util to purchase insurance against a low-likelihood ambiguous loss of 8 utils and another util to purchase a lottery ticket

\footnotetext{
${ }^{38}$ See the discussions of Klibanoff, Marinacci, and Mukerji (2005, pp. 1866, 1876) and Grant and Polak (2013).

${ }^{39} \mathrm{In}$ this sense it is intuitively similar to a preference for positive versus negative skewness.

${ }^{40}$ In each case, increasing $U\left(x_{i j}\right)$ by $\Delta U$ for all $i, j$ implies that $W(\cdot)$ will increase by $\Delta U$.
} 
Friedman-SaVage Insurance/Lottery Problem

\begin{tabular}{|c|c|c|c|c|c|c|c|c|}
\hline & \multicolumn{2}{|c|}{1 ball } & \multicolumn{2}{|c|}{1 ball } & \multicolumn{2}{|c|}{1 ball } & \multicolumn{2}{|c|}{1 ball } \\
\hline & Black & White & Red & Green & Blue & Yellow & Purple & Amber \\
\hline Bet I & 0 & 8 & 8 & 8 & 8 & 8 & 8 & 8 \\
\hline Bet II & 6 & 6 & 6 & 6 & 6 & 6 & 6 & 14 \\
\hline
\end{tabular}

offering a low-likelihood ambiguous gain of 8 utils, can go from Bet I to Bet II. Since these bets have the same mapping from (the 16) states to their conditional expected utility values, ${ }^{41}$ the Subjective Expected Utility Model would be exactly indifferent between them. But for that same reason so would each of the four major models, which suggests that correcting for attitudes toward objective risk, none can depart from SEU in the direction of a Friedman-Savage (1948)-type aversion to ambiguity in low-likelihood disasters coupled with a preference for ambiguity in low-likelihood high-stakes gains. $\frac{42}{}$

\section{Conclusion}

The examples of this paper show that a world of three or more possible outcome values allows for aspects of ambiguity and ambiguity aversion which cannot appear in the classic two-outcome Ellsberg examples, and are not fully captured by the major models of ambiguity aversion developed in response to these original examples. Three such aspects have been presented in this paper:

- The Thermometer Problem of Section III demonstrates that in a world of three or more outcomes, decision makers can exhibit Allais-type preferences over completely subjective prospects, which cannot be expressed in models whose preferences over almost-objective acts asymptote to Objective Expected Utility.

- The Slightly-Bent Coin and Meteorite Problems of Section IV demonstrate that in a world of three or more outcomes, prospects involving different sources and/or different degrees of ambiguity can have symmetric and/or identical good-news events, and thus cannot be strictly ranked by models which evaluate prospects solely through these events.

- The Ambiguity at Low versus High Outcome Levels Problems of Section V demonstrate that in a world of three or more outcomes, prospects with ambiguity at different outcome levels can have the same statewise conditional expected utility values, and hence cannot be strictly ranked by models which evaluate the objective uncertainty in mixed prospects solely through these statewise values.

This paper has considered four models of ambiguity aversion, each of which is subject to one or more of the above difficulties. There do, however, exist functional

\footnotetext{
${ }^{41}$ After the informationally-symmetric reordering of the payoffs $\{6,6,6,6,6,6,6,14\}$ to $\{6,14,6,6,6,6,6,6\}$ in Bet II.

${ }^{42}$ In particular, since the two bets have the same mapping from states to expected utility values, the Smooth Ambiguity Preferences form (10) cannot exhibit a strict preference in either direction even for an S-shaped or inverse S-shaped $\phi(\cdot)$ function.
} 
forms general enough to accommodate these and other aspects of ambiguity aversion, such as the Two-Stage Recursive Model of Segal (1987). ${ }^{43}$ As always, generalizations of a model (in this case, of Subjective Expected Utility) move us along the trade-off between the ability to accommodate specific paradoxes/examples and predictive power for other economic decisions. The proper point on this trade-off will, as always, depend on the findings of experimenters, the results of theorists and the application at hand.

\section{APPENDIX: \\ What is the Proper State Space for an Ellsberg Urn?}

As noted above, there is more than one way to specify the underlying state space of an Ellsberg urn. ${ }^{44}$ In our discussion of the classic Three-Color Paradox with its 60 balls of unknown color, we specified a 61-element space, with each state corresponding to the number of black balls. In our Section V discussion of the Three-Color Ambiguity at Low versus High Outcomes Problem, we specified a four-element space of the form $\{B B, B W, W B, W W\}$-had we instead defined states by the number of black balls, we would have had a 3 -element state space $\{0$ black balls, 1 black ball, 2 black balls\}. The relationship between the two specifications is that the latter space is slightly coarser that the former, with its state " 1 black ball" being the union of the states $B W$ and $W B$.

Does the choice of specification matter? Not always-given informational symmetry, the bets in the Two-Urn and Four-Color Ellsberg Paradoxes are such that the lessons of these examples would be the same under either specification.

However, the two specifications can yield different predictions over other types of bets, even under informational symmetry. Consider, as does Epstein (2010, p. 2088), bets on the actual composition of an Ellsberg urn-say Urn I in our initial Ambiguity at Low versus High Outcomes example. Informational symmetry over the space $\{B B, B W, W B, W W\}$ would lead to indifference between staking a prize on the two balls being the same color versus the two balls being of different colors. On the other hand, informational symmetry over the space $\{0$ black balls, 1 black ball, 2 black balls $\}$ would lead to a strict preference for the same-color bet. This distinction is magnified when applied to Ellsberg's 90- and 100-ball urns.

Whether individuals treat the state space $\{B B, B W, W B, W W\}$ or the state space $\{0$ black balls, 1 black ball, 2 black balls $\}$ as informationally symmetric is ultimately an empirical question. It turns out that the distinction does not matter for our analysis of this example. As mentioned, we adopted the state space $\{B B, B W, W B$, $W W\}$ in our analysis. However, if we instead adopt the space $\{0$ black balls, 1 black

\footnotetext{
${ }^{43}$ See Dillenberger and Segal (2015). Other models which can accommodate some or all of the examples in this paper and/or Machina (2009) are the Vector Expected Utility Model of Siniscalchi (2008b, 2009); the Uncertainty Averse Preferences Model of Cerreia-Vioglio et al. (2011); the Quantum Model of Aerts, Sozzo, and Tapia (2012); the Expected Uncertain Utility model of Gul and Pesendorfer (2013); and the models of Sagi (2007), Chew and Sagi (2008), Lehrer (2008, 2009, 2012), Abdellaoui et al. (2011), and Dumav and Stinchcombe (2013). See also the discussions of Baillon, L'Haridon, and Placido (2011, section IV), Blavatskyy (2013), and Dominiak and Lefort (2014).

${ }^{44}$ See also the discussions in Wakker (2010, sections 4.9 and 10.7) and Machina (2011).
} 


\begin{tabular}{lccc} 
& 0 black balls & 1 black ball & 2 black balls \\
\hline Urn I & $\left(\$ c, \frac{2}{3} ; \$ 100, \frac{1}{3}\right)$ & $\left(\$ 0, \frac{1}{3} ; \$ c, \frac{1}{3} ; \$ 100, \frac{1}{3}\right)$ & $\left(\$ 0, \frac{2}{3} ; \$ 100, \frac{1}{3}\right)$ \\
Urn II & $\left(\$ 0, \frac{1}{3} ; \$ 100, \frac{2}{3}\right)$ & $\left(\$ 0, \frac{1}{3} ; \$ c, \frac{1}{3} ; \$ 100, \frac{1}{3}\right)$ & $\left(\$ 0, \frac{1}{3} ; \$ c, \frac{2}{3}\right)$ \\
Expected utility & $\frac{2}{3}$ & $\frac{1}{3}$ & $\frac{1}{2}$ \\
\hline
\end{tabular}

ball, 2 black balls\}, the table preceding equations (27) through (30) would take the form in the above table, and the equations would take the following forms:

\section{Multiple-Priors Model:}

$$
\begin{aligned}
W_{M P}(\mathrm{Urn} \mathrm{I}) & =\underset{\left(p_{\text {Oblack }}, p_{1 \text { black }}, p_{2 \text { black })} \in \Pi\right.}{\min }\left[\frac{2}{3} \cdot p_{\text {0black }}+\frac{1}{2} \cdot p_{1 \text { black }}+\frac{1}{3} \cdot p_{2 \text { black }}\right] \\
& =W_{M P}(\text { Urn II }) .
\end{aligned}
$$

Rank-Dependent Model:

$$
\begin{aligned}
W_{R D}(\text { Urn I })=\frac{2}{3} & \cdot C(0 \text { black })+\frac{1}{2} \cdot[C(0 \text { black } \cup 1 \text { black })-C(0 \text { black })] \\
& +\frac{1}{3} \cdot[1-C(0 \text { black } \cup 1 \text { black })]=W_{R D}(\text { Urn II }) .
\end{aligned}
$$

Smooth Ambiguity Preferences Model:

$(29 *) \quad W_{S M}($ Urn I $)$

$$
\begin{aligned}
& =\int \phi\left(\frac{2}{3} \cdot p_{0 \text { black }}+\frac{1}{2} \cdot p_{1 \text { black }}+\frac{1}{3} \cdot p_{2 \text { black }}\right) \cdot d \mu\left(p_{\text {0black }}, p_{1 \text { black }}, p_{\text {2black }}\right) \\
& =W_{S M}(\mathrm{Urn} \text { II }) .
\end{aligned}
$$

Variational Preferences Model:

$(30 *) W_{V P}(\operatorname{Urn} \mathrm{I})$

$$
\begin{aligned}
& =\min _{\substack{\left(p_{\text {oblack }} p_{\text {lback }}, p_{\text {black }}\right) \\
\in \Pi}}\left[\frac{2}{3} \cdot p_{0 \text { black }}+\frac{1}{2} \cdot p_{1 \text { black }}+\frac{1}{3} \cdot p_{2 \text { black }}+\eta\left(p_{\text {0black }}, p_{1 \text { black }}, p_{2 \text { black }}\right)\right] \\
& =W_{V P}(\mathrm{Urn} \mathrm{II}) .
\end{aligned}
$$

In other words, the two urns will continue to have the same mapping from states to expected utility levels, and the four models will continue to imply indifference. Similar arguments apply to the other high- versus low-outcome examples of Section V. 


\section{REFERENCES}

Abdellaoui, Mohammed, Aurélien Baillon, Lætitia Placido, and Peter P. Wakker. 2011. "The Rich Domain of Uncertainty: Source Functions and Their Experimental Implementation.” American Economic Review 101 (2): 695-723.

Aerts, Diederik, Sandro Sozzo, and Jocylyn Tapia. 2012. "A Quantum Model for the Ellsberg and Machina Paradoxes." In Quantum Interaction: 6th International Symposium, QI 2012, Paris, France, June 27-29, 2012. Revised Selected Papers Lecture Notes in Computer Science, edited by Jerome R. Busemeyer, François Dubois, Ariane Lambert-Mogilansky, and Massimo Melucci, 48-59. Berlin: Springer-Velag.

Al-Najjar, Nabil, and Jonathan Lewis Weinstein. 2009. “The Ambiguity Literature: A Critical Assessment." Economics and Philosophy 25 (3): 249-84.

-Anscombe, Francis J., and Robert J. Aumann. 1963. “A Definition of Subjective Probability.” Annals of Mathematical Statistics 34 (1): 199-205.

- Baillon, Aurélian, Olivier L'Haridon, and Lætitia Placido. 2011. "Ambiguity Models and the Machina Paradoxes." American Economic Review 101 (4): 1547-60.

- Blavatskyy, Pavlo R. 2013. "Two Examples of Ambiguity Aversion.” Economics Letters 118 (1): 206-8.

Camerer, Colin. 1995. "Individual Decision Making.” In Handbook of Experimental Economics, edited by John H. Kagel and Alvin E. Roth, 587-703. Princeton: Princeton University Press.

-Camerer, Colin, and Martin Weber. 1992. "Recent Developments in Modeling Preferences: Uncertainty and Ambiguity." Journal of Risk and Uncertainty 5 (4): 325-70.

-Carlier, Guillaume, and Rose-Anne Dana. 2003. "Core of Convex Distortions of a Probability." Journal of Economic Theory 113 (2): 199-222.

Cerreia-Vioglio, Simone, Fabio Maccheroni, Massimo Marinacci, and Luigi Montrucchio. 2011. "Uncertainty Averse Preferences." Journal of Economic Theory 146 (4): 1275-330.

Chamberlain, Gary. 2001. "Minimax Estimation and Forecasting in a Stationary Autoregression Model." American Economic Review 91 (2): 55-9.

Chateauneuf, Alain. 1991. "On the Use of Capacities in Modeling Uncertainty Aversion and Risk Aversion.” Journal of Mathematical Economics 20 (4): 343-69.

Chateauneuf, Alain. 1994. "Modeling Attitudes Towards Uncertainty and Risk Through the Use of Choquet Integral." Annals of Operations Research 52 (1): 1-20.

-Chew, Soo Hong, and Jacob S. Sagi. 2008. "Small Worlds: Modeling Attitudes toward Sources of Uncertainty." Journal of Economic Theory 139 (1): 1-24.

Chipman, John S. 1958. "Stochastic Choice and Subjective Probability." Econometrica 26 (4): 613.

Chipman, John S. 1960. "Stochastic Choice and Subjective Probability." In Decisions, Values and Groups, edited by Dorothy Willner, 70-95. New York: Elsevier, Inc.

Diecidue, Enrico, Peter P. Wakker, and Marcel Zeelenberg. 2007. "Eliciting Decision Weights by Adapting de Finetti's Betting-Odds Method to Prospect Theory." Journal of Risk and Uncertainty 34 (3): 179-99.

Dillenberger, David, and Uzi Segal. 2015. "Recursive Ambiguity and Machina's Examples.” International Economic Review 56 (1).

Dominiak, Adam, and Jean-Phillipe Lefort. 2014. "Ambiguity Aversion and Machina's Paradoxes - A Reappraisal." Unpublished.

Dumav, Martin, and Maxwell B. Stinchcombe. 2013. "The von Neumann-Morgenstern Approach to Ambiguity." Unpublished.

-Ellsberg, Daniel. 1961. "Risk, Ambiguity, and the Savage Axioms." Quarterly Journal of Economics 75 (4): 643-69.

Ellsberg, Daniel. 1962. "Risk, Ambiguity, and Decision.” PhD diss., Harvard University.

Ellsberg, Daniel. 2001. Risk, Ambiguity and Decision. New York: Garland Publishing, Inc.

- Ellsberg, Daniel, Mark J. Machina, Klaus Ritzberger, and Nicholas Yannelis, eds. 2011. "Symposium on the 50th Anniversary of the Ellsberg Paradox." Economic Theory 48 (2-3): 219-548.

-Epstein, Larry G. 2001. "Sharing Ambiguity." American Economic Review 91 (2): 45-50.

-Epstein, Larry G. 2010. "A Paradox for the 'Smooth Ambiguity' Model of Preferences." Econometrica 78 (6): 2085-99.

Epstein, Larry G., and Martin Schneider. 2010. "Ambiguity and Asset Markets." Annual Review of Financial Economics 2 (1): 315-46.

-Ergin, Haluk, and Faruk Gül. 2009. “A Theory of Subjective Compound Lotteries.” Journal of Economic Theory 144 (3): 899-929.

Etner, Johanna, Meglena Jeleva, and Jean-Marc Tallon. 2012. "Decision Theory under Ambiguity." Journal of Economic Surveys 26 (2): 234-70. 
Fennema, Hein, and Peter P. Wakker. 1996. "A Test of Rank-Dependent Utility in the Context of Ambiguity.” Journal of Risk and Uncertainty 13 (1): 19-35.

-Friedman, Milton, and Leonard J. Savage. 1948. "The Utility Analysis of Choices Involving Risk." Journal of Political Economy 56 (4): 279-304.

Gilboa, Itzhak. 1987. "Expected Utility with Purely Subjective Non-Additive Probabilities." Journal of Mathematical Economics 16 (1): 65-88.

Gilboa, Itzhak, ed. 2004. Uncertainty in Economic Theory: Essays in Honor of David Schmeidler's 65th Birthday. London: Routledge Publishers.

Gilboa, Itzhak, and Massimo Marinacci. 2013. "Ambiguity and the Bayesian Paradigm." In Advances in Economics and Econometrics: Tenth World Congress. Vol. 1, Economic Theory, edited by Daron Acemoglu, Manuel Arellano, and Eddie Dekel, 179-242. Cambridge: Cambridge University Press.

Gilboa, Itzhak, and David Schmeidler. 1989. "Maxmin Expected Utility with a Non-Unique Prior." Journal of Mathematical Economics 18 (2): 141-53.

Grant, Simon, and Ben Polak. 2013. "Mean-Dispersion Preferences and Constant Absolute Ambiguity Aversion.” Journal of Economic Theory 148 (4): 1361-98.

Gül, Faruk, and Wolfgang Pesendorfer. 2013. "Expected Uncertain Utility and Subjective Sources." Unpublished.

Hagen, Ole. 1973. “Testing av nytteforventningshypotesen.” Sosialфkonomen 5: 9-18.

Hagen, Ole. 1979. "A Positive Theory of Preferences under Risk." In Expected Utility Hypotheses and the Allais Paradox, edited by Maurice Allais and Ole Hagen, 271-302. Dordrecht: D. Reidel Publishing Co.

Hansen, Lars Peter, and Thomas J. Sargent. 2001. "Robust Control and Model Uncertainty.” American Economic Review 91 (2): 60-6.

Hey, John, Gianna Lotito, and Anna Maffioletti. 2010. "The Descriptive and Predictive Adequacy of Theories of Decision Making under Uncertainty/Ambiguity." Journal of Risk and Uncertainty $41(2): 81-111$.

Kahneman, Daniel, and Amos Tversky. 1979. "Prospect Theory: An Analysis of Decision under Risk." Econometrica 47 (2): 263-91.

- Kelsey, David, and John Quiggin. 1992. “Theories of Choice under Ignorance and Uncertainty.” Journal of Economic Surveys 6 (2): 133-53.

Keynes, John Maynard. 1921. A Treatise on Probability. London: Macmillan and Company, Ltd.

Klibanoff, Peter. 2001. "Stochastically Independent Randomization and Uncertainty Aversion." Economic Theory 18: 605-20.

Klibanoff, Peter, Massimo Marinacci, and Sujoy Mukerji. 2005. "A Smooth Model of Decision Making under Ambiguity." Econometrica 73 (6): 1849-92.

Klibanoff, Peter, Massimo Marinacci, and Sujoy Mukerji. 2012. "On the Smooth Ambiguity Model: A Reply." Econometrica 80 (3): 1303-21.

Knight, Frank H. 1921. Risk, Uncertainty, and Profit. Boston: Houghton Mifflin.

Lehrer, Ehud. 2008. "A Comment on Two Examples by Machina.” Unpublished.

Lehrer, Ehud. 2009. “A New Integral for Capacities.” Economic Theory 39 (1): 157-76.

Lehrer, Ehud. 2012. "Partially-Specified Probabilities: Decision and Games." American Economic Journal: Microeconomics 4 (1): 70-100.

L'Haridon, Olivier, and Lætitia Placido. 2010. "Betting on Machina's Reflection Example: An Experiment on Ambiguity." Theory and Decision 69 (3): 375-93.

Lo, Kin Chung. 2008. "Risk, Ambiguity, and the Klibanoff Axioms." Economics Bulletin 4 (27): 1-5.

Luce, R. Duncan, and Howard Raiffa. 1957. Games and Decisions: Introduction and Critical Survey. New York: John Wiley and Sons.

Maccheroni, Fabio, Massimo Marinacci, and Aldo Rustichini. 2006. "Ambiguity Aversion, Robustness, and the Variational Representation of Preferences." Econometrica 74 (6): 1447-98.

MacCrimmon, Kenneth R., and Stig Larsson. 1979. "Utility Theory: Axioms versus 'Paradoxes'." In Expected Utility Hypotheses and the Allais Paradox, edited by Maurice Allais and Ole Hagen, 333-409. Dordrecht: D. Reidel Publishing Co.

Machina, Mark J. 1987. "Choice Under Uncertainty: Problems Solved and Unsolved." Journal of Economic Perspectives 1 (1): 121-54.

Machina, Mark J. 2004. “Almost-Objective Uncertainty.” Economic Theory 24 (1): 1-54.

Machina, Mark J. 2005. “ 'Expected Utility/Subjective Probability’ Analysis without the Sure-Thing Principle or Probabilistic Sophistication.” Economic Theory 26 (1): 1-62.

Machina, Mark J. 2009. "Risk, Ambiguity, and the Rank-Dependence Axioms." American Economic Review 99 (1): 385-92.

Machina, Mark J. 2011. "Event-Separability in the Ellsberg Urn.” Economic Theory 48 (2): 425-36. 
Machina, Mark J., and David Schmeidler. 1992. "A More Robust Definition of Subjective Probability." Econometrica 60 (4): 745-80.

Machina, Mark J., and Marciano Siniscalchi. 2014. “Ambiguity and Ambiguity Aversion.” In Handbook of the Economics of Uncertainty. Vol.1, edited by Mark J. Machina and W. Kip Viscusi, 729-807. Amsterdam: Elsevier B.V.

Mukerji, Sujoy, and Jean-Marc Tallon. 2004. "An Overview of Economic Applications of David Schmeidler's Models of Decision Making under Uncertainty." In Uncertainty in Economic Theory: Essays in Honor of David Schmeidler's 65th Birthday, edited by Itzhak Gilboa, 283-302. London: Routledge Publishers.

Nau, Robert F. 2001. "Uncertainty Aversion with Second-Order Probabilities and Utilities." Paper presented at the 2nd International Symposium on Imprecise Probabilities and Their Applications. Ithaca, New York.

Nau, Robert F. 2006. "Uncertainty Aversion with Second-Order Utilities and Probabilities." Management Science 52 (1): 136-45.

Poincaré, Henri. 1912. Calcul des Probabilités. 2nd ed. Paris: Gauthiers-Villars.

Quiggin, John. 1982. "A Theory of Anticipated Utility.” Journal of Economic Behavior and Organization 3 (4): 323-43.

Sagi, Jacob. 2007. "Modeling Implications of Source-Invariance to Machina's 'Almost-Objective Fair Bets'.' Unpublished.

Savage, Leonard J. 1972. The Foundations of Statistics. New York: Dover Publications. (1954).

-Schmeidler, David. 1986. "Integral Representation without Additivity." Proceedings of the American Mathematical Society 97 (2): 255-61.

-Schmeidler, David. 1989. "Subjective Probability and Expected Utility without Additivity." Econometrica 57 (3): 571-87.

-Segal, Uzi. 1987. “The Ellsberg Paradox and Risk Aversion: An Anticipated Utility Approach.” International Economic Review 28 (1): 175-202.

Segal, Uzi. 1990. “Two-Stage Lotteries Without the Reduction Axiom.” Econometrica 58 (2): 349-77.

Siniscalchi, Marciano. 2008a. "Ambiguity and Ambiguity Aversion." In The New Palgrave Dictionary of Economics. 2nd ed., edited by Steven N. Durlauf and Lawrence E. Blume, 138-42. London: Palgrave Macmillan.

Siniscalchi, Marciano. 2008b. "Machina's Reflection Example and VEU Preferences: A Very Short Note." Unpublished.

-Siniscalchi, Marciano. 2009. "Vector Expected Utility and Attitudes toward Variation." Econometrica 77 (3): 801-55.

Tversky, Amos, and Daniel Kahneman. 1992. "Advances in Prospect Theory: Cumulative Representation of Uncertainty." Journal of Risk and Uncertainty 5 (4): 297-323.

-Wakker, Peter P. 1990. "Under Stochastic Dominance Choquet-Expected Utility and Anticipated Utility are Identical.” Theory and Decision 29 (2): 119-32.

Wakker, Peter P. 2010. Prospect Theory for Risk and Ambiguity. Cambridge: Cambridge University Press.

-Wu, George, and Richard Gonzalez. 1999. "Nonlinear Decision Weights in Choice under Uncertainty." Management Science 45 (1): 74-85.

Zhang, Jiankang. 2002. "Subjective Ambiguity, Expected Utility and Choquet Expected Utility." Economic Theory 20 (1): 159-81. 
This article has been cited by:

1. David Dillenberger, Uzi Segal. 2015. RECURSIVE AMBIGUITY AND MACHINA'S EXAMPLES.

International Economic Review 56:10.1111/iere.2015.56.issue-1, 55-61. [CrossRef] 